\title{
Chapter 12 \\ Coordinating Distributed Energy \\ Resources During Microgrid Emergency \\ Operation
}

\author{
C. Gouveia, D. Rua, C. L. Moreira and J. A. Peças Lopes
}

\begin{abstract}
The development of the Smart Grid (SG) concept is the pathway for assuring flexible, reliable and efficient distribution networks while integrating high shares of Distributed Energy Resources (DER): renewable energy based generation, distributed storage and controllable loads such as Electric Vehicles (EV). Within the SG paradigm, the Microgrid (MG) can be regarded as a highly flexible and controllable Low Voltage (LV) cell, which is able to decentralize the distribution management and control system while providing additional controllability and observability. A network of controllers interconnected by a communication system ensures the management and control of the LV microgrid, enabling both interconnected and autonomous operation modes. This new distribution operation philosophy is in line with the SG paradigm, since it improves the security and reliability of the system, being able to tackle the technical challenges resulting from the large scale integration of DER and provide the adequate framework to fully integrate SG new players such as the EV. By exploiting the MG operational flexibility and controllability, this chapter aims to provide an extended overview on MG self-healing capabilities, namely on its ability of operating autonomously from the main grid and perform local service restoration. The MG hierarchical management and control structure is revisited and adapted in order to exploit the flexibility of SG new players, like the EV and flexible loads and integrate smart
\end{abstract}

C. Gouveia · D. Rua · C. L. Moreira $(\bowtie) \cdot$ J. A. Peças Lopes

INESC TEC-INESC Technology and Science,

Rua Dr. Roberto Frias, 378 4200-465 Porto, Portugal

e-mail: cmoreira@inescporto.pt

C. Gouveia

e-mail: cstg@inescporto.pt

D. Rua

e-mail: drua@inescporto.pt

J. A. Peças Lopes

e-mail: jpl@fe.up.pt 
metering infrastructures. The implementation of the MG architecture and communication infrastructure in a laboratorial facility is also presented and used to validate the MG self-healing capabilities.

Keywords Electric vehicle - Demand response - Frequency control • Islanding operation • Microgeneration - Microgrids • Smart grid

\subsection{Introduction}

The Microgrid (MG) is considered to be a Low Voltage (LV) distribution grid incorporating local generation, storage devices and responsive loads [1-3]. Through the future change of paradigm in the mobility sector, Electric Vehicles (EV) will become another resource to be integrated in the MG system, which can behave both as flexible loads or as mobile energy storage devices [4, 5]. In order to be flexible and controllable, the MG power infrastructure is supported by a communication and information system constituting the MG technical management and control system [1-3, 6, 7]. The local intelligence is responsible for coordinating and controlling local resources and enable innovative self-healing operating strategies [8].

MG applications can be divided in two major types:

- The MG operates as a Medium Voltage (MV) customer, being the main operational objective to meet the consumer's technical and economic requirements, while being capable of providing services to the upstream grid. This concept can be applied to a diversity of commercial, industrial or even military consumers.

- The MG is implemented as an extension of the distribution network management system. In this case, the MG main operational objective is to ensure the technical management and control of the LV network, when considering the integration of large amounts of microgeneration technologies, EV and responsive loads. However, the microgeneration, EV charging and loads control options will be limited by commercial agreements between the consumers and the electricity providers [5].

In the majority of the applications referred above, reliability is considered a major benefit of the MG system due to its ability to operate both interconnected to the main grid or autonomously as a physical island-emergency operation. In fact, case a disturbance occurs in the upstream MV network or in case of planned actions, the MG can be transferred to islanded operating conditions, supplying local loads with the MG generation capacity supported by fast acting storage units $[3,6]$. However, if the islanding fails or a general blackout occurs, the MG flexibility also allows performing local service restoration exploiting locally available generation and energy storage units [8]. Ensuring a successful autonomous operation is quite challenging, since the sudden islanding of the MG may cause high 
unbalances between local load and generation, which have to be compensated by fast acting storage devices in coordination with the microgeneration units and load shedding mechanisms [4, 9-11].

The fact that EV are expected to be connected to the LV network during long periods of time for charging purposes along with the predictability of its power consumption, makes them good candidates for the development of dedicated load control strategies. When regarded as flexible loads or distributed storage, they can contribute to increase the global MG storage capacity [4, 5]. Nevertheless, the increase of regulation capacity will depend on the number of EV connected to the system and on the state of charge of their batteries.

Complementing EV control strategies with innovative emergency demand response schemes for MG islanded operation will improve the security of operation during emergency conditions and minimize the consumers discomfort during outages [9-14]. Under such cases, innovative control functionalities need to be developed in order to promote an adequate coordination of the available resources.

The roll out of smart meters with bidirectional communication capabilities will provide a set of valuable measurements essential to perform an adequate analysis of the MG operating conditions and identify the most appropriate actions during MG emergency operation, including islanding and restoration procedures. Updating the emergency operation strategies according to the actual operating state of the MG will allow a better coordination of the available resources, consequently improving the MG resilience and success of the autonomous operation [14]. However, the new functionalities to be developed have to take into consideration the limitation of different communication solutions and technologies currently available, namely the use of private or public networks.

The feasibility of the MG concept integrating EV has been the focus of several research projects around the world. Following the track record on MG research, significant demonstration activities have been exploited worldwide [15-20], dedicated to the three main areas of knowledge involved in the MG operation, namely power systems, power electronics and information and communication technologies. Within this line, INESC Porto has been implementing a MG laboratorial infrastructure in order to exploit distinctive control and management solutions for key DER such as EV, loads and Renewable Energy Sources (RES) based microgeneration [20]. The laboratorial infrastructure aims to demonstrate the feasibility of the MG operation both in interconnected and autonomous mode while exploiting the specificities of different communication technological solutions that can be envisioned for a SG environment.

\subsection{MG Architecture and Communication Infrastructure}

The basic architecture of a MG consists in a hierarchical structure composed by a network of local controllers which are connected to each MG element and are headed by a central manager agent installed at the MV/LV substation-the MG 


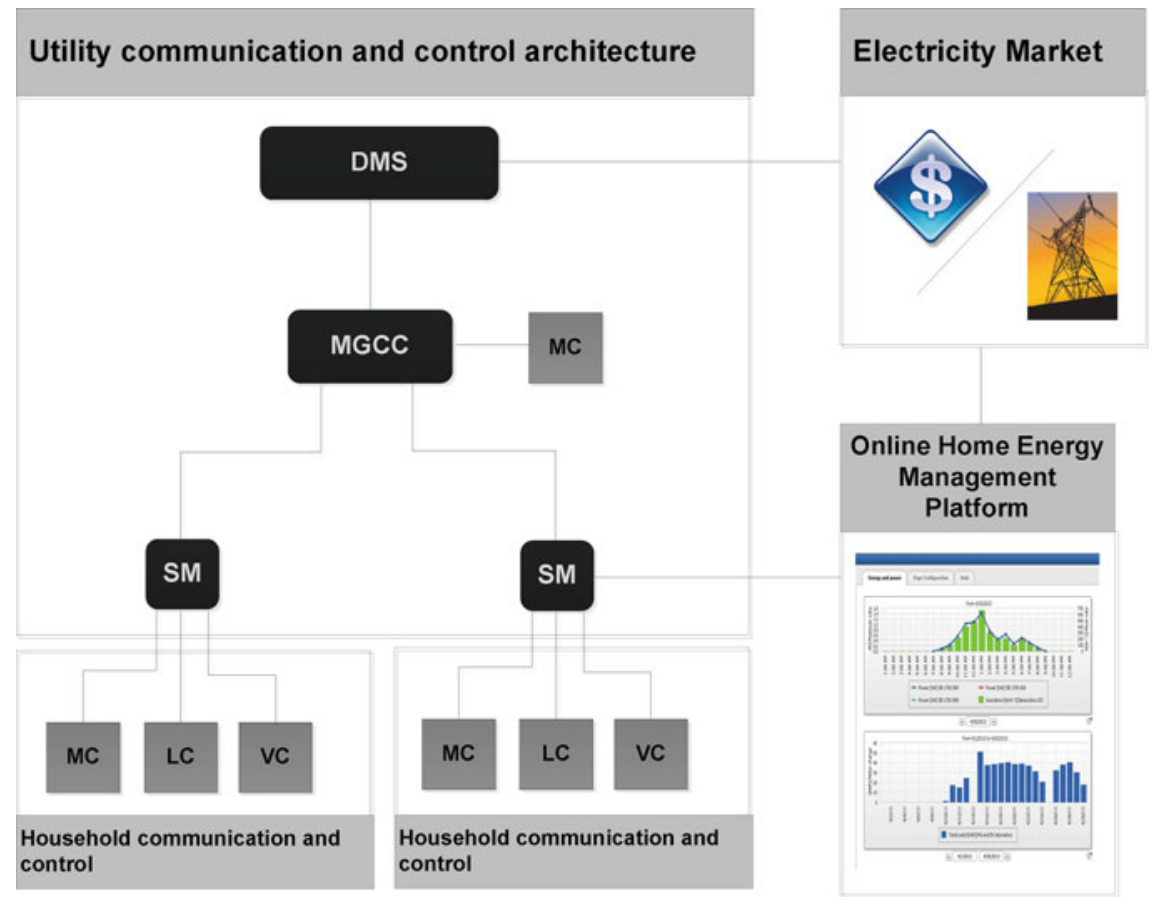

Fig. 12.1 Smart distribution network architecture based on MG concept

Central Controller (MGCC). Given the different characteristics of the MG resources, three types of local controllers can be defined: the Microsource Controller (MC), EV Controller (VC) and the Load Controller (LC) [3-5]. As shown in Fig. 12.1, the MG architecture can be easily adapted to integrate smart metering infrastructures as well as the associated processing capabilities to manage the data collected from the meters. At the consumer premises, the Smart Meter (SM) is expected to support bidirectional communications between the local controllers and the MGCC. Acting also as a local gateway it is able to aggregate the information related with power consumption, generation, service interruptions, voltage and other relevant data.

Communications systems and technologies have long been developed to support a diverse number of applications, many of them related with electric power system. However, a particular interest has been recently raised with the introduction of the SG concept, which intends, among others, to support enhanced control strategies in the distribution grids. This new distribution system paradigm introduces challenges for communications systems, namely because in these networks the need for information exchange, was until recently, very modest.

Different reference models and architectures have been defined, namely by NIST, IEEE and by the European Standards Organizations (ESO) with the purpose of establishing the main actors, data networks and domains of applications. 
Transversal to all of them is the highlighted importance of distribution and customer domains where the need of incorporating a considerable number of different potentially participating players is higher. There are also different functional and logical models that have been recently defined within the context of smart grids, establishing different information flows and requirement to be met to support specific types of information exchange.

\subsubsection{Smart Grid Communication Technologies and their Applicability to MG Operation}

A considerable amount of communication technologies that suite the needs of different applications targeted for SG is available. However, economical aspects along with technical limitation factors are among the main reasons for the use of particular technologies in detriment of others. The candidate technologies for the electric distribution networks can be associated with the use of public or private network. They can be typically distinguished between wired and wireless technologies.

The use of public communications network is commonly associated with telecommunication operators that make use of their infrastructure, based for instance on copper, fiber optics, or cellular networks, to provide services to their customers. The use of such networks is regarded by electric system operators with some scepticism due to the limitations that telecommunication operators have in guaranteeing the security, availability and reliability indexes usually associated with the electric sector.

The deployment of private networks in the back-haul or backbone of utilities has been very common, using several technologies in order to meet the different requirements associated with the applications to support. The use of these types of networks is therefore also regarded as preferred solution for the more demanding scenarios and applications, making way for the use of public network in less restrictive cases.

The potential for wired technologies envisioned by utilities are somehow narrowed down to the use of power lines as a communications medium, mainly due to the technical and economic constraints of deploying a cabled alternative, like copper or fiber. This has led to the exploitation of wireless alternatives compatible with the SG context.

\subsubsection{Power Line Technologies}

Power Line Communication (PLC) technologies explore the use of electric power lines as a medium to convey a bidirectional data exchange. In recent years, SG activities, in-building communications and home automation have brought a lot of 
attention to PLC as an alternative to unwanted or impractical wiring to setup communication networks. Power lines are inherently exposed to time-frequency varying noise, unmatched loads and interference from electrical or communication devices. The fact that in electric distribution networks power lines vary from grounded to overhead lines introduces further challenges in using this type of medium to exchange data. National legislation in different countries has introduced different limits in the transmission power and accepted frequency bands. For these reasons a large number of PLC technologies have been developed, each one addressing different applications and thus targeting different throughput values, frequency bands and channel access mechanisms. Implementations of PLC can be globally divided into narrow and broadband versions.

\section{Narrowband PLC}

The Narrowband PLC (NB-PLC) is usually associated with low and high speed versions. The latter is also sometimes referred to as medium speed to distinguish it from the broadband implementations. Different transmission frequency ranges are defined as well as a maximum transmission power in each range that must be respected either by a standard or by the legal disposition of each country. In Europe CENELEC is the entity responsible for the standardization and regulation of NB-PLC, whereas in the USA the Federal Communications Commission (FCC) and in Japan the Association of Radio Industries and Businesses (ARIB) are the legally assigned entities [21].

The first generation of NB-PLC made use of a single or double band carrier transmission schemes with simple modulation schemes like Phase-Shift Keying (PSK) and Frequency Shift Keying (FSK) to achieve a bandwidth of a few kbps, targeting remote metering applications. The second generation of PLC is associated with G3 and PRIME technologies, which were developed by different consortia with the purpose of supporting smart metering communication scenarios. Despite the use of Orthogonal Frequency-Division Multiplexing (OFDM) modulation schemes in G3 and PRIME, subtle physical layer details make them to differ slightly [22]. As a general rule, PRIME achieves higher data rates while G3 incorporates more powerful error correction coding for higher reliability of information. Another similar variation, G.hem, was introduced by the International Telecommunication Union (ITU) sharing some features with the previous two.

The IEEE 1901.2 [23] is an emerging narrowband standard under development since 2009. Defined as a low frequency OFDM narrowband for SG applications, it is set to use frequencies below $500 \mathrm{kHz}$ and data rates up to $500 \mathrm{kbps}$ supporting both indoors and outdoors communications. In the outdoors context this standard targets MV and LV distribution feeders. It is expected that coexistence mechanisms are defined to enable the interaction with G3 and PRIME systems. 


\section{Broadband PLC}

The initial developments of Broadband PLC (BB-PLC), also known as Broadband Powerline (BPL), were started in the in-building environment, namely as a replacement for conventional Ethernet infrastructure as an alternative, or complementary solution for wireless solutions. In the last years BB-PLC has been explored as a communication infrastructure outside the building context, namely as a candidate technology for last-mile communications. Wide frequency bands, generally in the range of 2-30 MHz, are used worldwide, except in Japan where BB-PLC is not permitted. Higher bandwidths are potentially available in some countries but they are generally bounded due to TV broadcast systems. One of the main promoters of BB-PLC has been the HomePlug Alliance that targeted domestic environments to implement this type of networks. A particular implementation of HomePlug standard was introduced in 2010 and designated as Green PHY (GP) for in-home automation as a support for SG applications. It is prepared to coexist with other HomePlug implementations.

The IEEE 1901 [24] is another BPL standardized implementation which uses some of the strategies defined in the HomePlug variants. Unlike the latter, it is designed to be deployed both inside and outside the building environment. Different PHY layers are defined along with different modulation schemes and error correction coding mechanisms. Some of the approaches used in IEEE 1901 are expected to be included in the IEEE 1901.2 NB-PLC.

The ITU has also defined a BPL standard designated G.hn, with the purpose of supporting SG applications such as Advanced Metering Infrastructure (AMI) and energy management including EV. It allows the use of power line communications inside buildings, as well as in the last-mile.

\subsubsection{Wireless Technologies}

Wireless communication systems are another type of technologies that can also be explored in the context of SG, either as an alternative or as a complementary solution. Some of the generally highlighted advantages of wireless technologies in this scope are the ease of installation, maintenance and modularity that allow a higher degree of flexibility. Another advantage of wireless implementations, when associated to battery systems, is the potential capability to continue operating despite the occurrence of disturbances that lead to the disconnection of parts of a feeder, thus compromising communications over power lines. The potential low cost is also pointed out as an advantage when well established and mature solutions are employed. Even so, there are some general challenges associated with the use of wireless technologies, namely the need for careful planning due to the adverse channel propagation conditions, the limitation in the use of free or licensed Radio Frequency (RF) bands, the presence of obstacles, and the shared nature of the communication medium. 


\section{IEEE 802.11/Wi-Fi}

One of the most known and disseminated IEEE standards is the 802.11 that defines the features of a wireless local area network as an alternative to cabled Ethernet. Its commercial designation, $\mathrm{Wi}-\mathrm{Fi}$, is promoted by the $\mathrm{Wi}-\mathrm{Fi}$ Alliance, which has contributed for the widespread availability and implementation of this technology, making it a ubiquitous solution used in different communication infrastructures. In SG its use has been explored inside and outside the building environment, in applications as varied as distribution substation automation, DER monitoring, control LAN, among others. The IEC 61850 defines some of the applications that can be support by Wi-Fi networks [25].

\section{IEEE 802.16/WiMAX}

The IEEE 802.16 was released by IEEE as a standard for wireless metropolitan area networks, targeting both Line of Sight (LOS) and non-LOS applications. The WiMAX is the designation of the wireless devices based on this standard. It is promoted by the WiMAX Forum that certified the compatibility and interoperability of the devices from different manufacturers. Although WiMAX was created with other type applications in mind, the fact is that SG applications like automatic meter reading, real-time pricing, outage detection and restoration are also targeted to be supported by WiMAX [25].

\section{IEEE 802.15.4/ZigBee}

A family of standards for wireless personal area networks was introduced by IEEE with the 802.15.4. It defines low power, low rate and low complexity networks to convey small amount of information over relatively small distances. The ZigBee stack is responsible for the design and implementation of applications over IEEE 802.15.4. It originally targeted applications such as building automation, embedded sensing or wireless sensor networks. Recently, SG applications like the control of domestic appliances or even direct load control by a system operator or utility, allowing smart meters to interact with a home area network manager, have been considered to be supported by this type of networks [26].

\section{Emerging Wireless Solutions and Opportunities}

The use of conventional wireless technologies, typically single-hop, for SG application has been subject to a general discussion and acceptance in specific scenarios. As an alternative, multi-hop technologies can also be considered in this context. One of such cases can be found in the recent IEEE 802.15.4 g, which defines a mesh network of flat smart meters to support smart metering. Nevertheless 

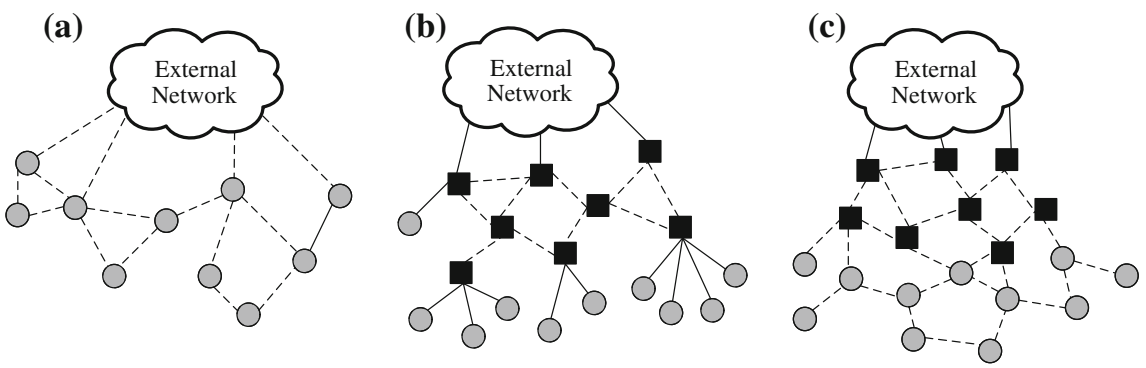

Station (STA)

Mesh Acces Point (MAP)

Fig. 12.2 Wireless mesh network topologies

this type of solution is not tailored for last-mile implementations, found in distribution networks, where node distances can vary significantly [27].

Given the characteristics of the distribution networks, wireless mesh networks can be used as a means to extend the coverage of a communications network allowing access to an infrastructure network that can be wired or wireless. As shown in Fig. 12.2, several topologies can be explored for this purpose that range from a completely flat network where all nodes can relay information to more complex and tiered approached where mesh access point nodes are responsible for relaying the information. Nevertheless, hybrid strategies can also be implemented.

Wireless Mesh Networks (WMN) can potentially offer higher levels of redundancy and robustness in terms of data communications, in the event of temporarily or permanent node or link loss. Routing algorithms can be adapted to dynamic network topologies to deal with channel varying conditions. However, the use of WMN typically aggravates some of the limitations of conventional single-hop wireless communications. Associated challenges like fairness [28], scheduling in terms of fair-sharing [29] or resource optimization [30] and cross-layer mechanisms like scheduling with congestion control are already addressed by the research community. The use of WMN in distribution networks is facilitated given the scope in which they have to operate. Most devices are not expected to have mobility characteristics and planning is made usually in a medium/long term perspective. Power constraints are very low and the use of battery support may allow the survival of such systems in the event of power failures or even in more severe cases where blackout occurs. The geographic span of last mile SG communication networks may not require a high number of mesh nodes, namely relays, to ensure a proper coverage. Besides, data aggregation, typically upstream, may be used to allow mode flexible scheduling schemes.

Solutions like Wi-Fi network Infrastructure eXtension (WiFIX) allow the extension of wired communication networks using simple and efficient WMN [31]. This solution is regarded as an alternative to the IEEE 802.11 s which defines mesh networking within the 802.11 family. The original WiFIX system has evolved [31,32] and it consists of a simple and efficient tree-based algorithm for 
stub WMN that runs over 802.11 legacy MAC. Being a system that runs above the MAC layer it can be implemented in wireless technologies other than IEEE 802.11, making it suitable of SG last-mile scenarios.

An opportunity for wireless communications in SG can be found in the disconnection of the analog TV broadcast services and consequent transition to digital terrestrial TV. This can potentially allow frequency bands to be available for SG applications. These are in particular desirable bands since they are associated with transmission ranges which are compatible with the last-mile segment. Even when using multi-hop solutions these frequencies could allow a similar level of coverage but with less number of hops. Within this RF reusability spirit, TV white spaces communications have also been considered as potential solution for the support of SG services, although current regulation restrictions limit the reuse of these bands [26].

\subsection{Microgrid Control and Emergency Functionalities}

The stability of the conventional power system relies in the regulation of the system's voltage and frequency, which is achieved by the control of large synchronous units. However, the majority of MG systems are inverted dominated being inertialess, due to the inexistence of rotating masses directly coupled to the grid [3].

MG components such as Microsource (MS), storage units and EV require a power electronic interface (DC/AC or AC/DC/AC) in order to be connected and exchange power with the AC distribution grid. The inverters control system is responsible for controlling the active and reactive power exchanged with the grid, ensure high power quality and grid synchronization functionalities. Besides, some additional services may be required by the grid operator, namely voltage and frequency regulation, harmonic compensation or even active filtering [33, 34]. The two most common control strategies used for the inverters connected to the power systems are [3, 6, 33]:

- PQ inverter control: the inverter operates by injecting a given active and reactive power set point into the grid. The control is implemented as a current-controlled voltage source, with the objective of exporting a controlled amount of power (active and reactive) to the grid (these inverters are usually referred as grid tied inverters).

- Voltage Source Inverter (VSI) control: the inverter is able to control the frequency and magnitude of its output voltage. A VSI, also referred as a grid forming unit, defines the grid voltage and frequency reference by assuring a fast response in order to continuously balance power generation and loads.

In interconnected mode, the MG voltage and frequency reference is given by the upstream network. In these operating conditions the inverters can be operated 


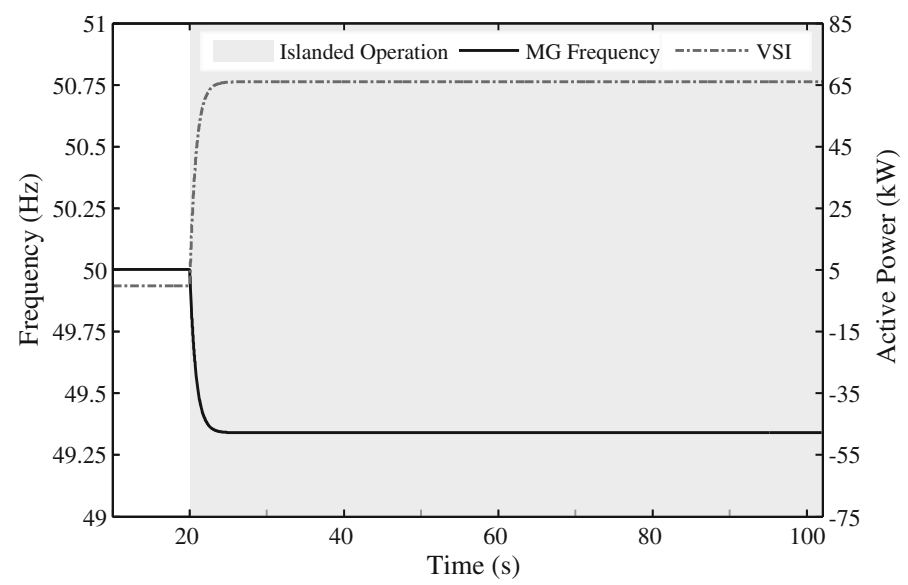

Fig. 12.3 SMO strategy: MG frequency and active power injected by storage unit

with a PQ control strategy, being the set-points for P and Q provided either by the MGCC or by other forms of local control. However, when the MG disconnects from the main grid, due to a fault upstream or a planned outage, it is necessary to establish the voltage in magnitude and frequency. Similarly to what has been defined for large power systems, the MG requires specific primary and secondary frequency control strategies, in order to maintain power balance between generation and loads and restore frequency to adequate values [3, 6]:

- Primary Control, which is performed locally to continuously assure the balance between load and generation.

- Secondary Control, which ensures voltage and frequency regulation in order to maintain these electrical variables within acceptable limits. This control level also includes the coordination of synchronization actions to reconnect the MG to the main grid after islanding operation. It is usually performed at the central controller such as the MGCC.

\subsubsection{Primary Voltage and Frequency Regulation}

In conventional power systems, synchronous generators share load variations in accordance with their droop characteristics. This general principle of conventional power systems can be applied to inverters in order to change its output frequency as a function of the output power. This principle is also important for parallel operation of variable frequency AC voltage sources, similarly to what happens in conventional power systems. In order to provide voltage and frequency regulation, the storage unit grid coupling inverter is controlled as a VSI device with external droop control loops, as in Eq. (12.1) [3, 6], 


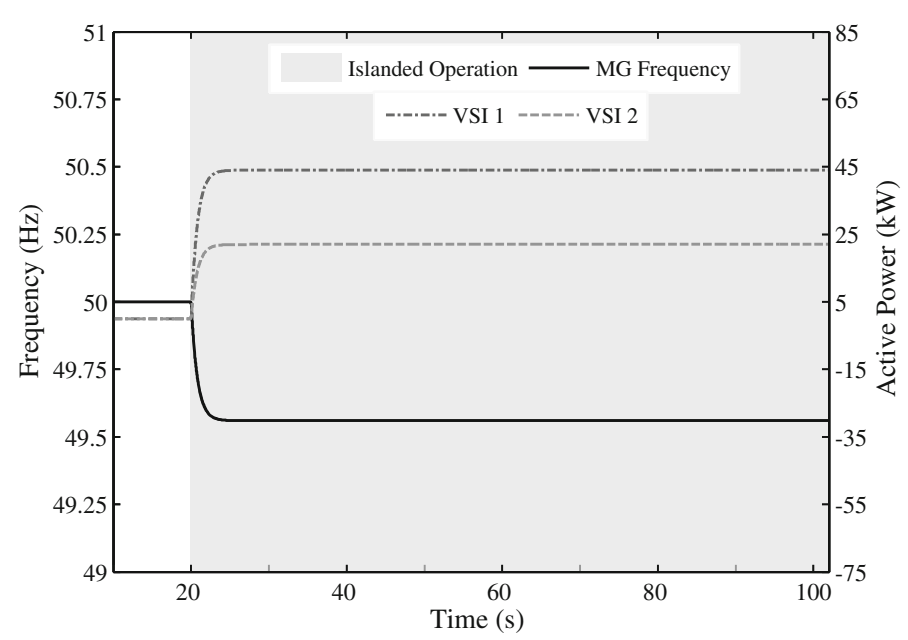

Fig. 12.4 MMO strategy: MG frequency and active power injected by the two storage units (VSI 1 and VSI 2)

$$
\begin{aligned}
& \omega=\omega_{\text {grid }}-k_{P} \times P \\
& V=V_{\text {grid }}-k_{Q} \times Q
\end{aligned}
$$

where $P$ and $Q$ are the inverter active and reactive power outputs, $k_{P}$ and $k_{Q}$ are the droop slopes (positive values) and $\omega_{0}$ and $V_{0}$ are the idle values of the angular frequency and voltage (at no load conditions).

Figure 12.3 shows a MG frequency response after an unplanned islanding at $\mathrm{t}=20 \mathrm{~s}$. The power unbalance between the MG generation and load will cause a frequency variation in accordance to the active power-frequency (P-f) droop, similarly to the response of the conventional synchronous machines. In this case the MG was importing about $66 \mathrm{~kW}$ prior to islanding, which was compensated by the storage unit. This control principle allows the VSI to react to system disturbances based only on information available at its terminals, not requiring fast communications between the MC controllers and the MGCC.

When the MG operates with a single VSI, the MG is said to operate in Single Master Operation (SMO) mode. However, the MG can operate with more than one VSI controlled through droop functions, in a Multi-Master Operation (MMO) strategy. In this case, when a disturbance occurs, the storage units will respond to the power imbalance and share the power variations according to their droop characteristics, as in Eq. (12.2).

$$
\Delta P=\sum_{i=1}^{n} \Delta P_{i}
$$

where $\Delta P$ is the unbalance between the MG generation and load and $\Delta P_{i}$ is the active power injected by the i-th VSI connected to the MG. 
Fig. 12.5 EV frequencydroop characteristic

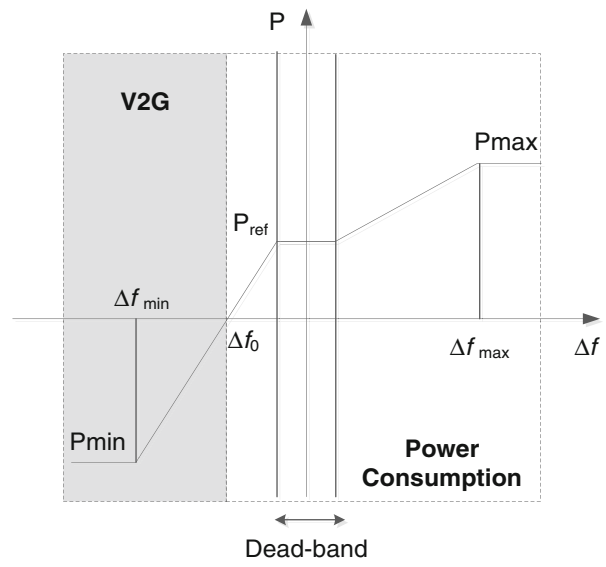

The MG frequency deviation and the power sharing among the VSI can be determined by the system of equations represented in Eq. (12.3).

$$
\left[\begin{array}{ccccc}
1 & k_{P 1} & 0 & \cdots & 0 \\
1 & 0 & k_{P 2} & \cdots & 0 \\
\ldots & \ldots & \ldots & \ddots & \vdots \\
1 & 0 & 0 & \cdots & k_{P n} \\
0 & 1 & 1 & \cdots & 1
\end{array}\right] \times\left[\begin{array}{r}
\omega^{\prime} \\
\Delta P_{1} \\
\Delta P_{2} \\
\vdots \\
\Delta P_{n}
\end{array}\right]=\left[\begin{array}{c}
\omega_{\text {grid }} \\
\omega_{\text {grid }} \\
\vdots \\
\omega_{\text {grid }} \\
\Delta P
\end{array}\right]
$$

where $\omega^{\prime}$ is the post-disturbance MG angular frequency and $\omega_{\text {grid }}$ is the predisturbance MG angular frequency.

Figure 12.4 shows the MG frequency response after an islanding when adopting an MMO strategy. The power disturbance is shared by the two units as a function of the storage unit capacity. In this case VSI 2 has half of VSI 1 capacity. Comparing to the SMO strategy in Fig. 12.3 the resulting frequency deviation is smaller, since the power injected by each unit is also lower. MMO strategy is more robust than $\mathrm{SMO}$, because the voltage and frequency control relies in several (distributed) VSI units [3, 6].

\subsubsection{EV Contribution to Primary Frequency Support}

The deployment of EV within the new mobility paradigm introduces significant power requirements for battery charging purposes which impacts the operation of the LV network by affecting voltage profiles and line flows. In order to mitigate the resulting impacts, the implementation of smart charging strategies has been shown to be an effective way of dealing with the mentioned problems. Additionally, during MG autonomous operation, EV can also be exploited in order to reduce the 


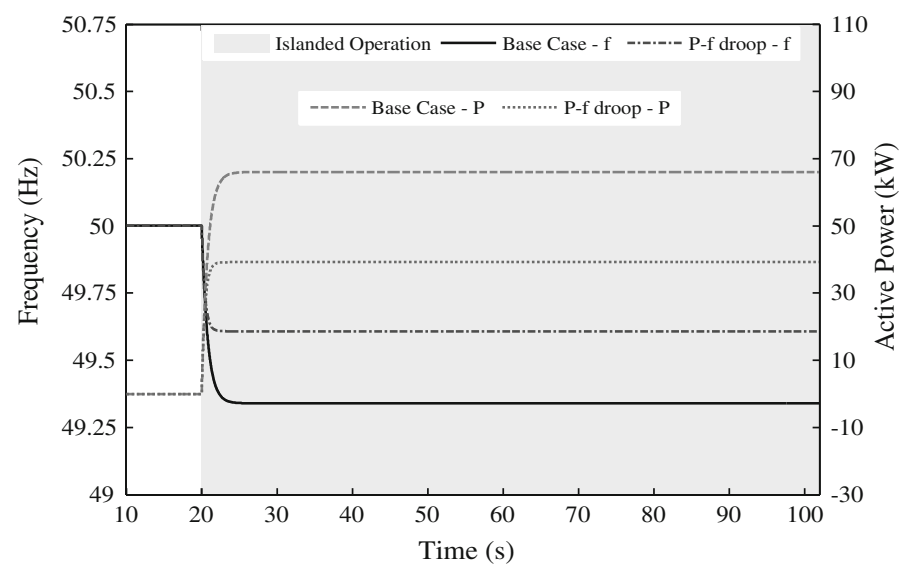

Fig. 12.6 MG islanding with EV control: MG frequency and VSI active power response

Fig. 12.7 MG frequency and EV active power response

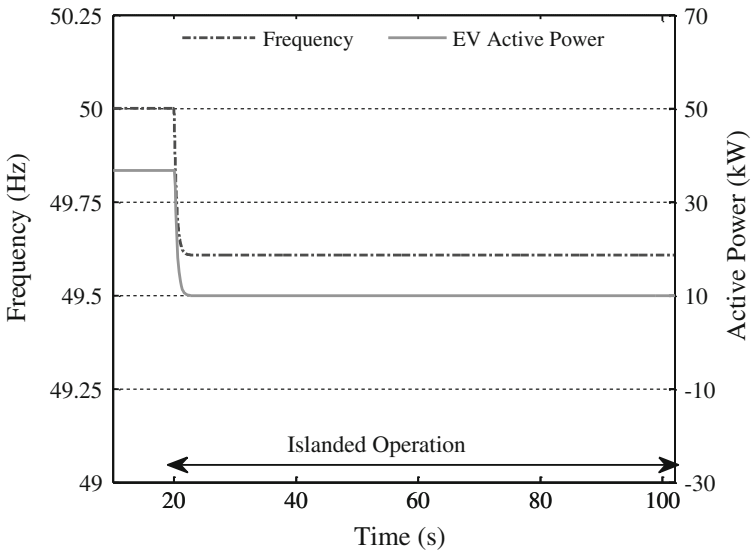

central storage capacity required to assure $\mathrm{MG}$ system robustness during islanding conditions $[4,5,14]$. A P- $f$ droop control strategy implemented at the EV charger has been proposed in $[4,5]$, where the EV will modify the power exchanged with the LV grid based on the MG frequency.

As shown in Fig. 12.5, for frequencies around the nominal value the EV will charge the battery at a pre-defined charging rate $\left(\mathrm{P}_{\text {ref }}\right)$. If a disturbance occurs and the frequency drops below the dead-band minimum, the EV reduces its power consumption, reducing the load of the system. When the MG frequency overpasses the frequency dead-band maximum, the EV can also increase its power consumption. For large disturbances, causing the frequency to go below the zero-crossing frequency $\left(f_{0}\right)$, the EV starts to inject power into the grid (Vehicle-to-Grid-V2G functionality). When the MG frequency becomes out of the pre-defined frequency range the vehicle will inject/absorb a fixed power $\left(\mathrm{P}_{\max }\right.$ or $\left.\mathrm{P}_{\min }\right)$. 
Fig. 12.8 Local secondary frequency control of PQ controlled MS

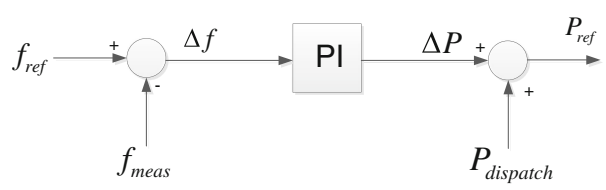

Fig. 12.9 Local secondary frequency control for VSI

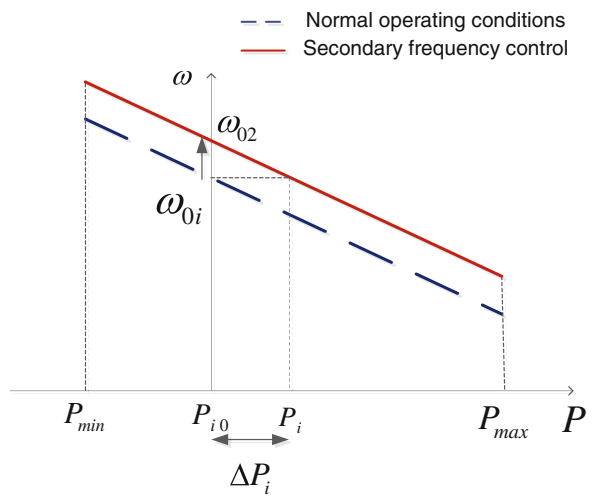
controlled MS

The definition of the EV control parameters will depend on the EV charger characteristics and on the willingness of EV owners to participate in such services. These parameters may differ from grid to grid and can be changed remotely by the MGCC in order to promote adequate coordination with the MG frequency regulation mechanisms (load shedding schemes, availability of energy storage devices and their state of charge).

Figure 12.6 compares the MG frequency and the VSI active power response after an islanding at $\mathrm{t}=20 \mathrm{~s}$ when a SMO control strategy is adopted. As shown in Fig. 12.7 the EV controlled through P-f droop have reduced their power consumption from 36.75 to $10 \mathrm{~kW}$, reducing the MG load and consequently the power injected by the VSI.

\subsubsection{Secondary Load-Frequency Control}

The secondary frequency control ensures that after a disturbance the frequency returns to its nominal value, by increasing the power output of controllable MS, such as microturbines and/or fuel cells. As shown in Figs. 12.3 to 12.6, during a transient the power balance is assured by the MG energy storage devices, which would keep on injecting or absorbing active power until the frequency is restored to the idle value. 


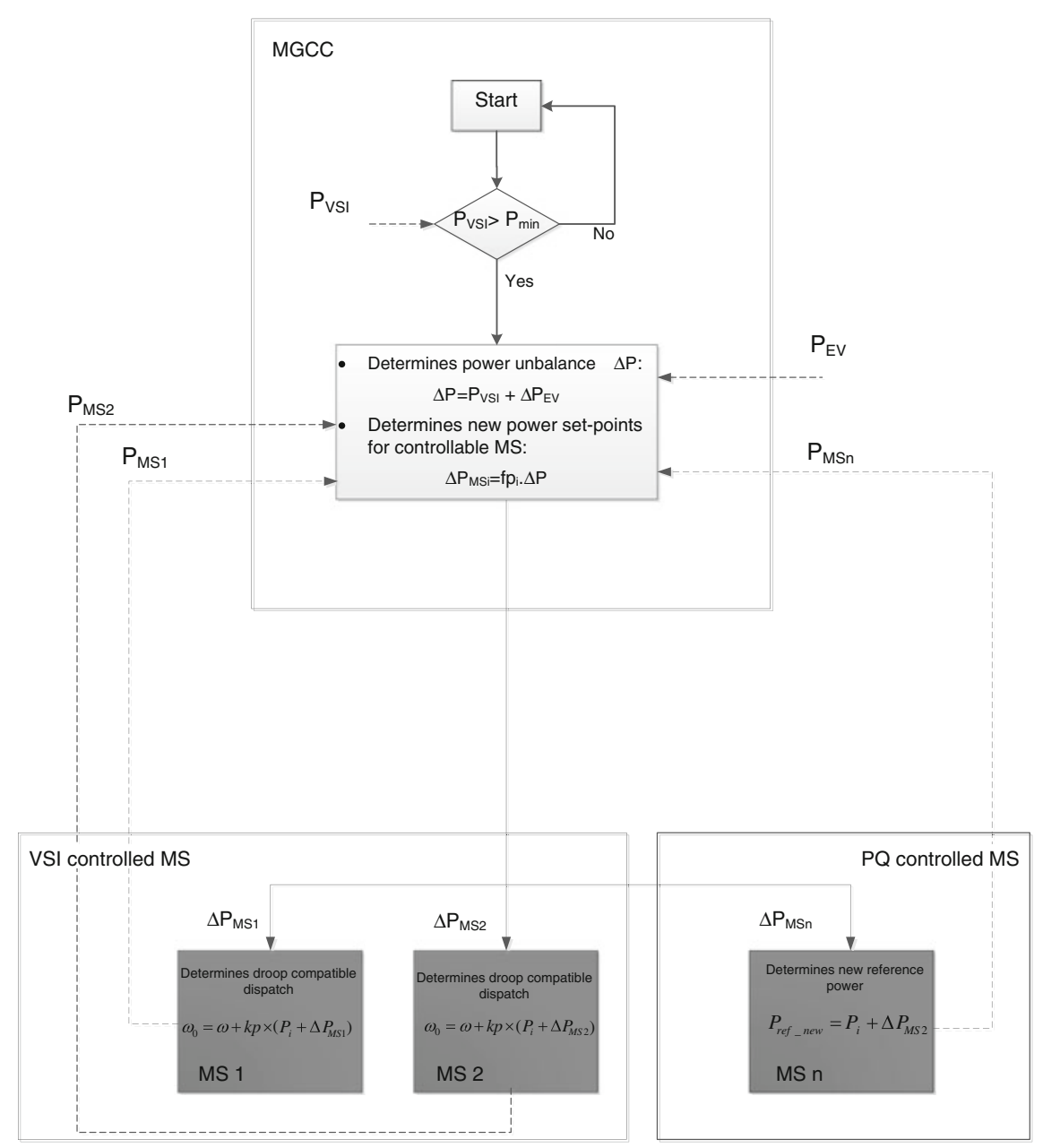

Fig. 12.10 Centralized secondary control algorithm

In [35] two secondary load-frequency control strategies are identified: one implemented locally at each controllable MS and another centralized and mastered by the MGCC. Local secondary control is implemented as an external control loop of the active power control of controllable MS, as shown in Fig. 12.8. If the MS inverter is controlled with a PQ strategy, the reference active power $\left(\mathrm{P}_{\mathrm{ref}}\right)$ to be injected by the MS is determined by a PI controller based on MG frequency error. If the MS inverter is controlled as VSI, local secondary control can be performed by updating the idle frequency $\omega_{0 \mathrm{i}}$ of the MS P- $f$ droop function, as represented in Fig. 12.9. As proposed in $[3,35]$ a PI controller can be used to determine the new idle frequency $\left(\omega_{02}\right)$ based on the MG frequency deviation in order to update the 
Fig. 12.11 MG frequency response considering: only primary regulation (Base Case), primary and secondary control and the EV P-f droop

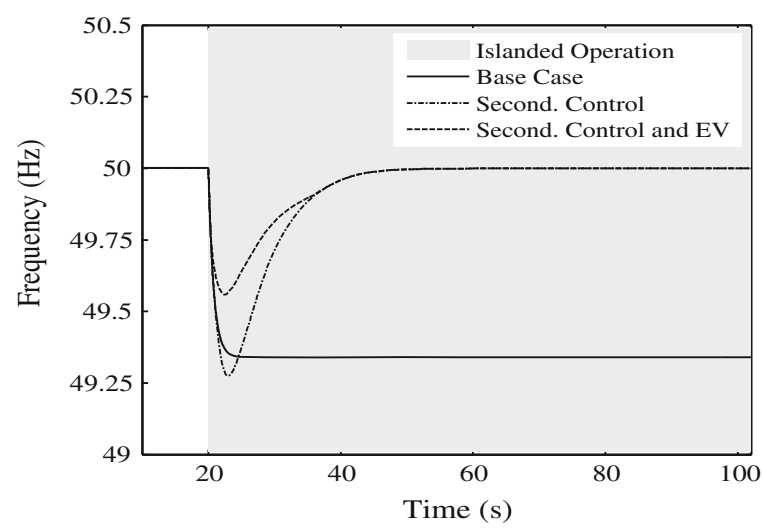

active power droop characteristic. The main advantage of local secondary frequency control is that it only relies on local measurement to define the new reference power. However, the MS active power response will also depend on the PI controller parameters.

Centralized secondary control determines the new MS active power set-points at the MGCC and then sends them to the respective local controllers. Based on the MG frequency deviation and on an economic dispatch algorithm, the MS new set-points are determined according to participation factors, which are based on pre-defined cost functions or on the MS capacity [35, 36].

Taking advantage of the data provided by the smart meters a centralized secondary control strategy based on active power unbalance can be designed. The algorithm shown in Fig. 12.10 is implemented at the MGCC and performs the MS power dispatch when the power injected by the storage unit installed at the MV/LV substation surpasses a defined limit.

As shown in Fig. 12.10, the MG power unbalance $(\Delta \mathrm{P})$ is determined based on the power injected by the $\mathrm{MG}$ main storage unit $\left(\mathrm{P}_{\mathrm{VSI}}\right)$ and on the EV injected power $\left(\mathrm{P}_{\mathrm{EV}}\right)$. The contribution of each controllable MS will be defined through a participation factor $\left(f_{p i}\right)$, which is determined (for unit $i$ ) by the ratio between its reserve and the total controllable MS reserve, as in Eq. (12.4),

$$
\left\{\begin{array}{l}
f p_{i}=\frac{R_{i}}{R} \\
\Delta P_{M S_{i}}=\Delta P \times f p_{i} \\
\sum_{i=1}^{n} \Delta P M S_{i}=\Delta P
\end{array}\right.
$$

where $\Delta P_{M S i}$ is the emergency active power step of unit $i, \Delta P$ is the active power unbalance following a disturbance and $R_{i}$ is the reserve capacity of unit $i$. The resulting set-points are then sent from the MGCC to local MC. In order to be 
Fig. 12.12 VSI active power response considering: only primary regulation (Base Case), primary and secondary control and the EV P-f droop
Fig. 12.13 Controllable MS power dispatch
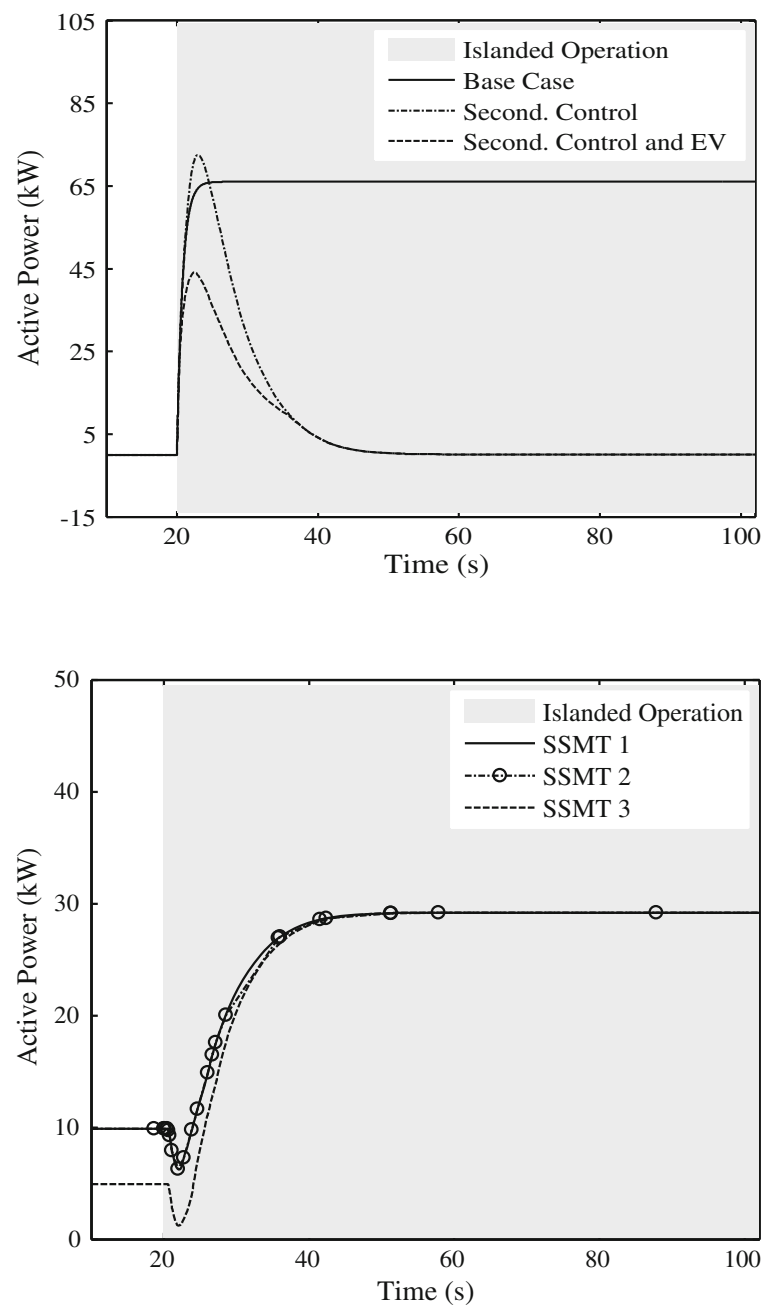

compatible with both SMO and MMO modes of operation, in the case of MS controlled as VSI, the algorithm determines new idle values for the angular frequency of the MS droop characteristics $\left(\omega_{0}\right)$, in order to obtain the desired power adjustment $\left(\Delta P_{M S i}\right)$. For PQ controlled MS, the new power set-point is directly sent to the MC.

Figure 12.11 compares the MG frequency when considering primary and secondary frequency response with and without the participation of EV through P- $f$ droop characteristic (MG islanding takes place at $\mathrm{t}=20 \mathrm{~s}$ ).

As shown in Figs. 12.12 and 12.13, secondary control reduces the power injected by the storage unit, recovering the frequency to the nominal value (in this case $50 \mathrm{~Hz}$ ). In this case, in order to compensate the $66 \mathrm{~kW}$ of power imported 
from the MV network, it was assumed that the MG comprises also three Single Shaft Microturbines (SSMT) running on gas that were dispatched to their maximum capacity (30 kW in this scenario) after islanding. The typical response of this type of units is presented in Fig. 12.13.

\subsubsection{Demand Response}

Controlling the MG loads may be required when operating autonomously for the two distinct situations:

- MG with insufficient generation reserve. In this case, the load will have to be curtailed until the system reconnects to the upstream network or the generation increases.

- MG with sufficient reserve. Although the MG has sufficient reserve, it may happen that, for a certain disturbance, the storage units State of Charge (SOC) is not able to ensure a secure operation or the frequency deviation expected for a given disturbance surpasses admissible limits. The implementation of a temporary load curtailment will help improve the MG frequency regulation capacity and reduce the solicitation from the storage unit(s).

The MG load shedding schemes are usually based on under-frequency or on rate-of-change-frequency relays [3]. However, if the MG has sufficient generation capacity, some demand response strategies may help dealing with transient disturbances resulting from load or variable generation, compensating the slow response of MS to power control signals.

In [13] an Adaptive Hill Climbing (AHC) load control method was proposed in order to improve the MG voltage and frequency regulation. The AHC determines the minimum percentage of responsive loads (electric water heaters) which should be disconnected/connected based on the MG frequency deviation. The commands are then sent to the electric water heaters controllers. The method was tested considering a small system with a diesel generator, which ensured secondary frequency control. Still, in the absence of synchronous machines, the MG frequency response will depend on the storage unit and controllable MS response to control signals.

Several frequency responsive load control methods based on local frequency measurements have been proposed in literature. The Frequency Adaptive Power Energy Reschedule (FAPER) method [10] proposes the control of consumers loads based on a local frequency measurement. In case of a sudden load increase or generation loss causing a severe frequency disturbances, the loads will be disconnected during the time of response of the secondary control. In [11], a Dynamic Demand Control (DDC) method is proposed in order to control refrigerators. An alternative controller to the conventional thermostat will connect/disconnect the refrigerator based on the frequency in addition to the freezer air temperature. The results presented have shown that the DDC method potentially improves power 
Fig. 12.14 Priority load shedding algorithm

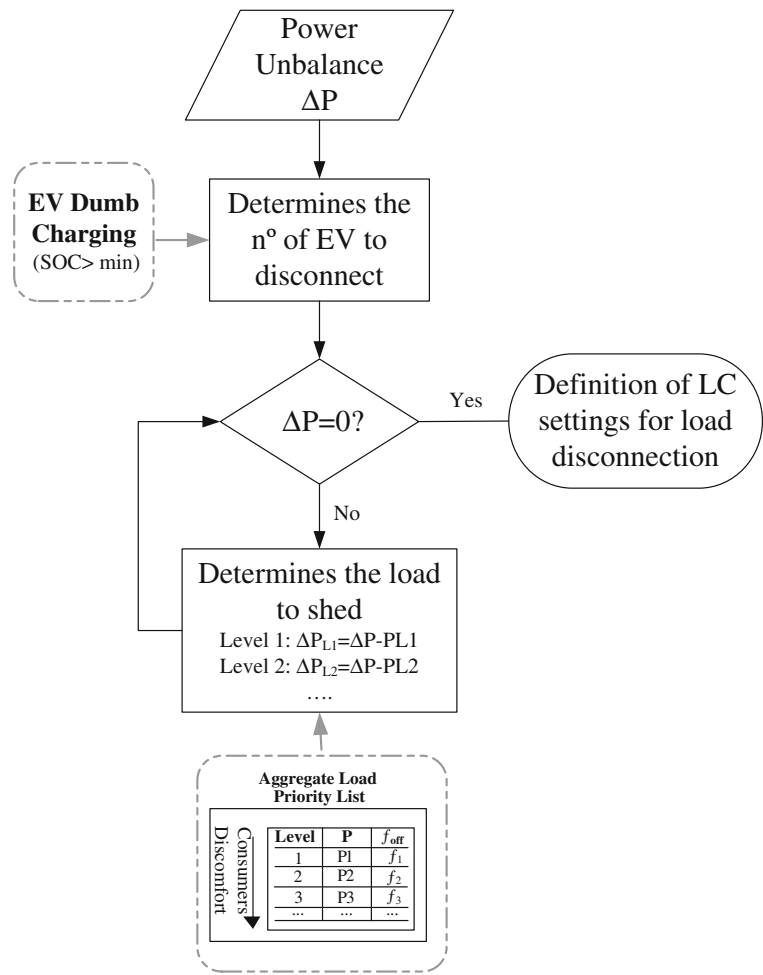

system stability, when facing the sudden loss of generation, increase of load or dealing with a large penetration of variable generation. In [12] a load reduction algorithm is proposed considering the local frequency measurement from smart meters. The load control method aggregates typical domestic appliances according to the level of discomfort caused by their disconnection.

Based on the frequency load control methods proposed in [10-12, 14] a selective load control scheme is proposed in order to control the MG loads, where the consumers will be able to prioritize the availability of its home appliances to participate in the MG load control scheme. At the MGCC an online tool periodically characterizes the MG operating state and evaluates the MG resilience in the event of a disturbance affecting MG load and generation balance. Based on the load priority list aggregated at the MGCC and on the amount of load which is required to be disconnected $(\Delta \mathrm{P})$, the load shedding algorithm shown in Fig. 12.14 defines a set of actions to be parameterized at the local control level, namely: load disconnection in LC, EV disconnection or droop parameterization at VC.

The activation/parameterization of the controls is performed previously to the disturbances. The MGCC will send to the SM a frequency threshold for the activation of the different load priority levels, which is then communicated to LC and VC. When there is available reserve capacity, load reconnection takes place 
Fig. 12.15 MG frequency response with EV control and demand response

Fig. 12.16 VSI active power output with EV control and demand response
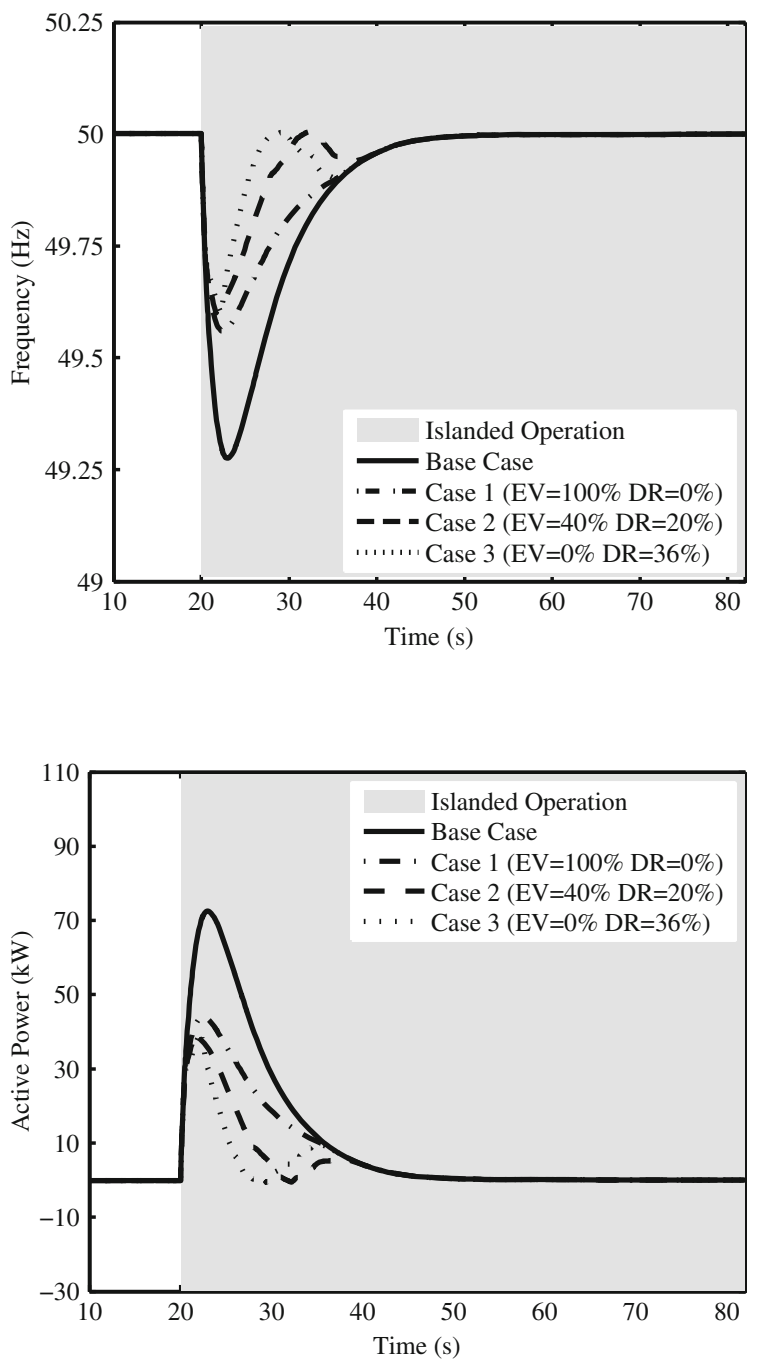

when the frequency recovers to a value close to the nominal one (for example, at least $49.9 \mathrm{~Hz}$ ). In order to avoid simultaneous load reconnections, a random time delay is considered for load reconnection after the moment frequency effectively reaches the pre-defined value.

Figure 12.15 shows the MG frequency behavior when the load participates in the MG frequency regulation following islanding at $t=20 \mathrm{~s}$. Four scenarios were tested: 
- Base Case-considering only primary and secondary frequency control. The EV and loads do not participate in the MG frequency regulation.

- Case 1-All the EV connected to the MG are controlled with P- $f$ droop, representing a total of $49 \mathrm{~kW}$. Load control is not considered.

- Case 2-Only $40 \%$ of the EV connected to the system are controlled with P-f droop characteristic and $20 \%$ of the MG load is available for participating in the demand response scheme.

- Case 3-The EV do not participate in the MG frequency regulation and $36 \%$ of the MG load is available for participating in an active demand response scheme.

The coordination of EV and load control strategies demonstrates great benefits in terms of the severity of the frequency disturbance and consequently of the storage solicitation. As shown in Fig. 12.16, similarly to the results obtained for the EV P-f droop control, the participation of load provides spinning reserve to the system during the secondary control response time. Further improvements on the reconnection of loads can be performed in order to minimize the transients.

\subsubsection{Managing the MG Energy Balance Following Islanding}

The MG frequency and voltage control mechanisms described in Sects. 12.3.1 and 12.3.3 ensure continuous power balancing and frequency restoration to nominal values during islanding operating conditions. However, the effectiveness of the strategies will depend on several operating conditions, such as:

- MG storage capacity, which is essential to ensure primary frequency regulation.

- Controllable MS reserve capacity, in order to perform secondary frequency regulation.

- Non-controllable MS power production, which acts as a negative load in the system.

- MG flexible load, including the EV connected to the LV network.

- MG load in comparison to total generation.

The information sent by the smart meters to the MGCC can be used to characterize the MG operating conditions and coordinate the available resources in order to improve the MG resilience to severe disturbances. Updating the MG emergency operation strategies according to the MG actual operating state will allow:

- Minimizing the amount of load to be disconnected.

- Minimizing the time during which loads are disconnected.

- Ensure that the MG has sufficient storage capacity to ensure primary frequency control following a given disturbance.

- Maintain frequency excursions within admissible limits. 
Fig. 12.17 Algorithm for defining the MG emergency operation strategy

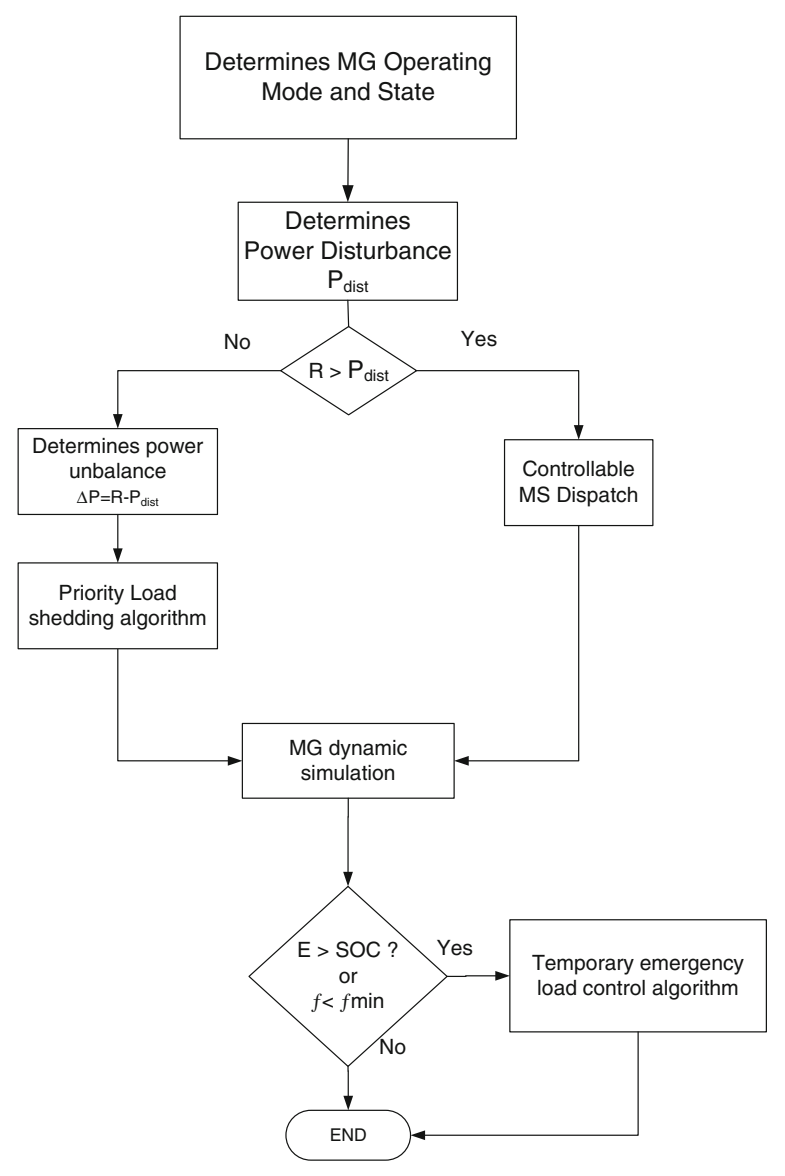

The algorithm presented in Fig. 12.17 will run online at the MGCC in order to analyze the MG operating conditions and then identify the most appropriate actions to ensure a secure islanding. It consists in four main steps, namely:

(1) Characterize the $M G$ operating state based on the information sent by smart meters. The algorithm determines the MG storage capacity, microgeneration reserve capacity, power flow between the MG and the MV network and load (divided between the controllable and non-controllable load).

(2) Determine the severity of the disturbance. If the MG is operating interconnected to the main grid, the occurrence of an unplanned islanding will cause an active power unbalance $\left(\mathrm{P}_{\text {dist }}\right)$ equal to the power exchanged with the $\mathrm{MV}$ network. If the MG is operating islanded, the power unbalance will result from the changes in loads or generation. It is important to notice that planned islanding events are not a key concern, since adequate control action can be taken in order to balance the MG load and generation prior to islanding, thus minimizing the associated transient phenomena. 


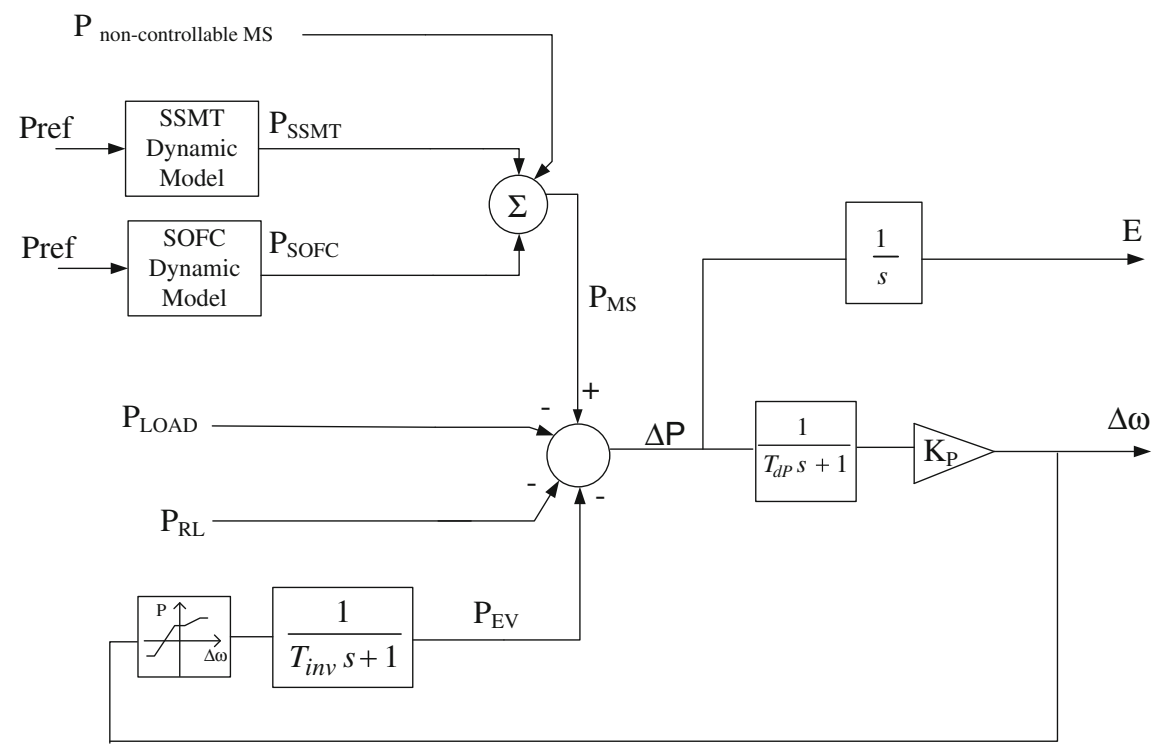

Fig. 12.18 MG simplified dynamic model to run at the MGCC

(3) Determine the amount of load to shed. In case the MG does not have enough reserve capacity, it is necessary to exploit emergency responsive loads and shed some part of the MG load in order to ensure power balance.

(4) Evaluate the security of the MG during an unplanned islanding. The algorithm determines if the MG has enough storage capacity to ensure power balance during the time required for restoring the frequency to nominal values or if the frequency goes out of admissible limits. Otherwise, it might be necessary to shed some load temporarily.

In Step (3) the amount of load to shed $\left(\Delta P_{\text {shed }}\right)$ is determined as in Eq. (12.5), based on the MG reserve capacity $(\mathrm{R})$ and on the active power unbalance $\left(P_{\text {dist }}\right)$ estimated in Step (2),

$$
\Delta P_{\text {shed }}=R-P_{\text {dist }}
$$

During islanding conditions, if the MG lacks energy to ensure power balance, the system will collapse. The energy balance in the storage unit $(E)$ can be determined by Eq. (12.6).

$$
E=E_{\Delta P}-E_{M S}-E_{V E}-E_{R L}
$$

where $E_{\Delta P}$ is the energy resultant from the power unbalance after the MG disturbance, $E_{M S}$ is the energy provided by the MS (both controllable and noncontrollable) and $E_{V E}$ is the energy resultant from the response of the EV to the frequency deviation. $E_{R L}$ results from: (1) the energy not supplied to responsive 
loads, which were disconnected due to the emergency state of the MG or (2) the energy to be supplied to dump loads, in case the disturbance leads to an excess of generation regarding the MG load.

Based on Eq. (12.6) it is possible to evaluate the energy required to ensure secure islanded operation. However, the energy injected/absorbed by the MG storage unit will depend on the response of the controllable MS such as SSMT or fuel cells (for example Solid Oxide Fuel Cells-SOFC). Since these units present a non-linear power response, the energy required to ensure MG robust operation following a disturbance cannot be accurately estimated using simplified linear models.

In order to overcome this difficulty, a simplified MG dynamic model that is represented in Fig. 12.18 was adopted. The model consists on a single equivalent bus, considering only the load, generation and storage resources. The model adopted neglects the presence of the LV network and the dynamics of the power converters, which are faster than the MG dynamic behavior. The storage unit and $\mathrm{EV}$ are represented by their external P- $f$ control loop. $T_{d P}$ is the delay related to the response of voltage source inverters and $T_{i n v}$ is the delay of the EV grid coupling inverter [13]. Loads and non-controllable MS are represented as constant active power.

The load shedding and the generation emergency dispatch are used as inputs of the MG dynamic model in order to evaluate the energy balance within the MG and the expected frequency deviation for a given period. As outputs, the model provides the total energy injected by the storage units and the MG frequency response. Based on these values, the algorithm then verifies if the MG storage units have sufficient capacity to ensure power balance and if the minimum frequency does not violate the admissible frequency limits $\left(f_{\mathrm{min}}\right)$. If these conditions are violated, it is possible to follow an iterative procedure to determine an additional amount of load to be disconnected and compensate the slow response of some MS to power control signals. The frequency threshold for its activation can also be identified, based on the frequency response obtained from the MG simplified model.

The proposed approach is intended to support MG islanding operation during short periods of time (i.e. less than $1 \mathrm{~h}$ ). For larger time frames of operation in islanding conditions, complementary approaches need to be considered, involving forecasting of loads with different degrees of flexibility (including EV), as well as forecasts for renewable based microgeneration.

\subsection{Microgrid Service Restoration Procedure}

The occurrence of general blackouts is a rare event but has severe economic and social impacts, as a consequence of the long restoration times resulting from complex procedures followed by system operators [37-39]. The unique characteristics of each electric power systems preclude the definition of general restoration plans. The restoration procedures usually consist on pre-established guidelines 
and operating routines, involving transmission and distribution operators. For each step, system operators have to coordinate generation and load to maintain system stability, ensure reactive power balance to keep system voltages within acceptable limits and limit switching transient voltages, amongst others [37-39].

When compared to conventional power system restoration, the MG Black Start (BS) procedure will benefit from a considerable problem size reduction, and hence the reduction of the number of controllable variables. However, as discussed previously, the MG is an inverter dominated grid, thus requiring very specific voltage and frequency regulation strategies exploring the MG energy storage and the MS generation capacity [8].

The MG restoration procedure is triggered by the MGCC when a general or local blackout occurs, or when the MV network is not able to restore MG operation after a pre-defined time interval. Similarly to conventional power systems, the MG restoration procedure consists on a sequence of actions to be checked and performed by MG local controllers in coordination with the MGCC. However, the procedure should be fully automatic without requiring the intervention of distribution network operators.

In order to perform service restoration at the MG level, it is assumed that the MG is equipped with [8]:

- MS with black start capability, capable of communicating to the MGCC their generation availability and operational status.

- LV switches to disconnect the MG feeders, loads and MS in case a generalized fault occurs.

- Communication infrastructure powered by dedicated auxiliary power units, in order to ensure the communication between the MGCC and the local controllers.

- Adequate protection equipment in order to protect MS and the LV grid from the fault currents and to isolate the faulted area. Since the BS procedure involves a step-by-step connection of MS to the LV grid, the short-circuit power at the point where protection devices are installed will change. Thus, under such protection strategy, it is assumed that during the restoration procedure the MGCC has the ability to change the protection devices settings in order to efficiently detect and isolate MG faults.

In order to provide BS capability, controllable MS such as SSMT and or fuel cells require additional DC storage capacity to power the ancillary equipment required when starting the MS. Also, in order to supply local loads, the MS coupling inverter has to be controlled as a VSI. Therefore, during the MG restoration procedure a Multi-Master Operation (MMO) strategy could be adopted increasing the MG robustness during the procedure. When the MG stabilizes before synchronizing to the upstream network, the MS grid-coupling inverters can switch its control strategy to PQ. 

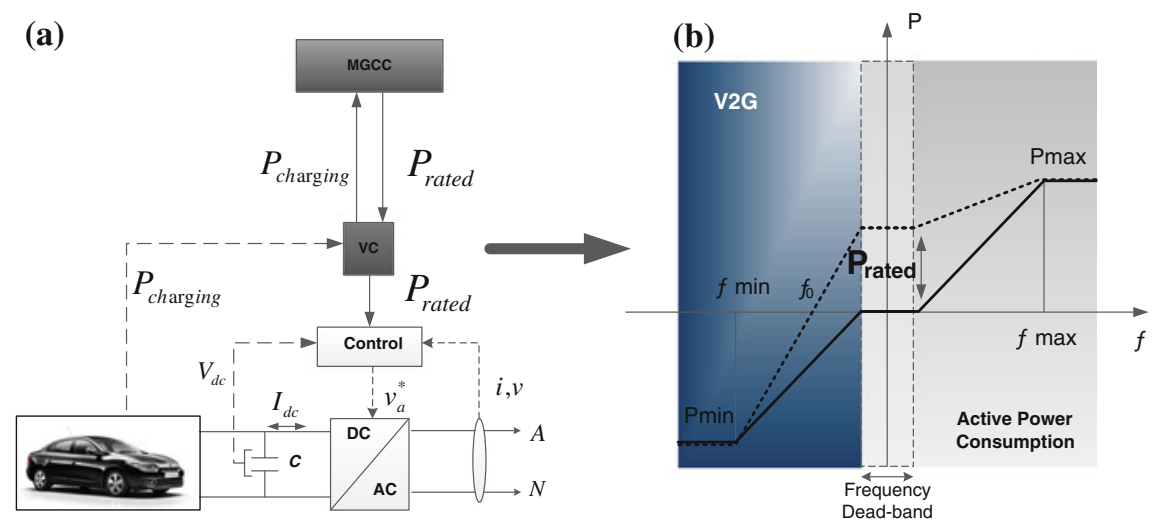

Fig. 12.19 EV control and interaction with the MGCC for the MG service restoration

\subsubsection{Participation of $E V$ in the MG Restoration Procedure}

The EV can be integrated in the MG restoration procedure as grid supporting units. The EV available to provide this service will be connected to the MG during the first steps of the restoration procedure. They won't be allowed to charge at the nominal frequency. Instead, the EV should be synchronized to the grid with a zero power set-point, as shown in Fig. 12.19. As a result, during the first steps of the procedure and whenever the frequency remains within the EV P-f droop frequency dead-band, the EV will not exchange power with the MG. However, when the loads and MS begin to be reconnected, the EV will inject or absorb power in order to reduce the resulting frequency disturbance, consequently reducing the solicitation from the main storage unit and providing frequency support to the MG.

\subsubsection{BlackStart Methodology}

After a general blackout, the MGCC will trigger the BS procedure, based on the information about the MG pre-disturbance load scenario. Following the MG restoration strategy proposed in [8], the overall procedure can be organized in the following sequence of events:

1. MG status determination. The MGCC evaluates the network status both upstream and downstream:

a. Upstream network status - the MGCC only activates the restoration procedure if there is no alternative to reconnect to the main grid. Before activating the procedure, the MGCC waits for a confirmation of the occurrence of local or 
general blackout from the distribution system operator, in order to eliminate the possibility of an interconnection switch malfunction, or possible reconnection to the main grid through MV network reconfiguration.

b. Downstream network status - the MGCC evaluates the LV network status, analyzing switches status and alarms, in order to check the existence of local faults or equipment failures. At this stage the MGCC also evaluates the generation and active load resources, in order to ensure the successful MG service restoration. Historical data resulting from the $\mathrm{MG}$ operation can provide information about the priority loads to be restored.

2. MG preparation in order to start the restoration procedure. The MGCC should send to the local controllers (MC, LC), a signal to ensure the disconnection of loads and MS from the LV network. Then, MS with local BS capability can be restarted and be used in order to power some of the local loads. This procedure ensures that the MS with storage capacity providing back power to their local loads are not energizing larger parts of the LV network.

3. MG energization, connecting the MG central storage unit at the MV/LV substation and closing the substation LV feeder switches. The connection of the storage unit in no load/generation conditions ensures that the MG is operating at nominal frequency and voltage.

4. Synchronization of the running MS to the MG. The synchronization is enabled by the MGCC, being the necessary conditions-such as phase sequence, frequency and voltage differences (both in phase and amplitude) checked by the MC, through synchro-check relays.

5. Connection of EV. The MGCC will send a control signal to all smart meters in order to connect the EV chargers with a zero power set-point and enable the P-f droop functionality.

6. Coordinated reconnection of loads and non-controllable MS, considering the available storage capacity and local generation, in order to avoid large frequency and voltage deviations during load and non-controllable generation connection.

7. Enable EV charging. After restoring priority loads and generation, if the MG remains with sufficient reserve capacity the MGCC will enable the EV charging. In order to avoid further disturbances, the MGCC will send new reference power to the smart meters and dispatch the controllable MS to meet the new load.

8. MG synchronization with the main grid, when the service is restored at the MV network. The MGCC should receive a confirmation from the distribution network operator to start the synchronization with the upstream network. The synchronous conditions are checked locally through a synchro-check relay. 


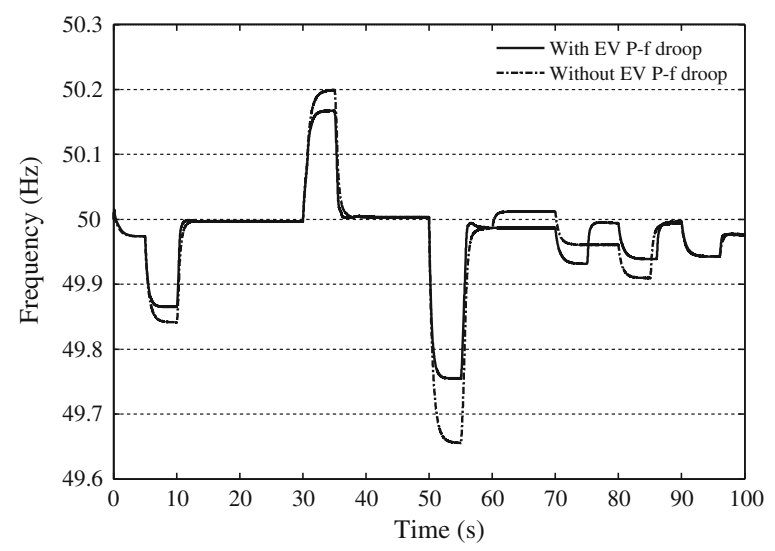

Fig. 12.20 MG frequency response during the MG rebuilding

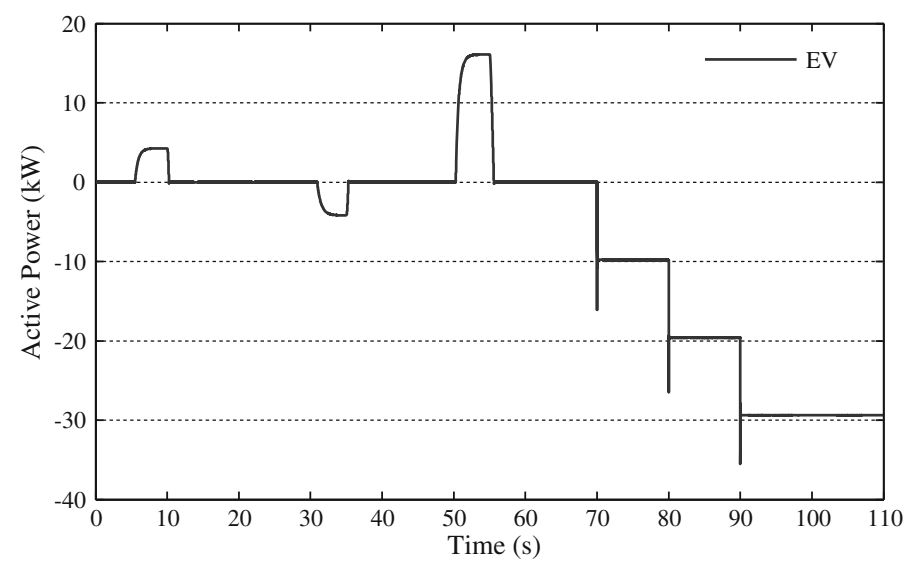

Fig. 12.21 EV total power output during MG rebuilding

\subsubsection{Analyzing the Benefits of EV Participation on the MG Rebuilding}

Figure 12.20 compares the MG frequency behavior during the reconnection of loads and non-controllable MS when considering both non-controlled charging and $\mathrm{V} 2 \mathrm{G}$ service. At $\mathrm{t}=5 \mathrm{~s}$ the MGCC enables the reconnection of the first load block causing a frequency deviation of $1.5 \mathrm{~Hz}$, which is then corrected by the secondary control by increasing the controllable generation output. The load reconnection is interleaved with the reconnection of the non-controllable MS at $t=30 \mathrm{~s}$, followed by the reconnection of the second block of loads at $t=50 \mathrm{~s}$. After the restoration 


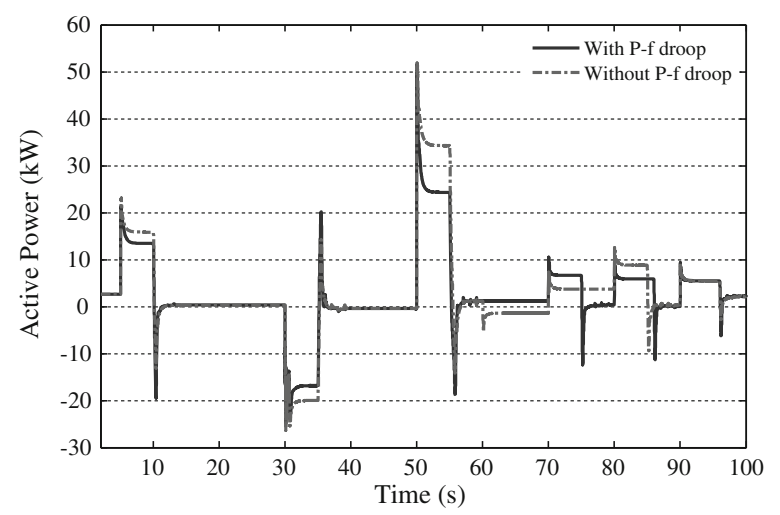

Fig. 12.22 Active power injected by the MG main storage unit

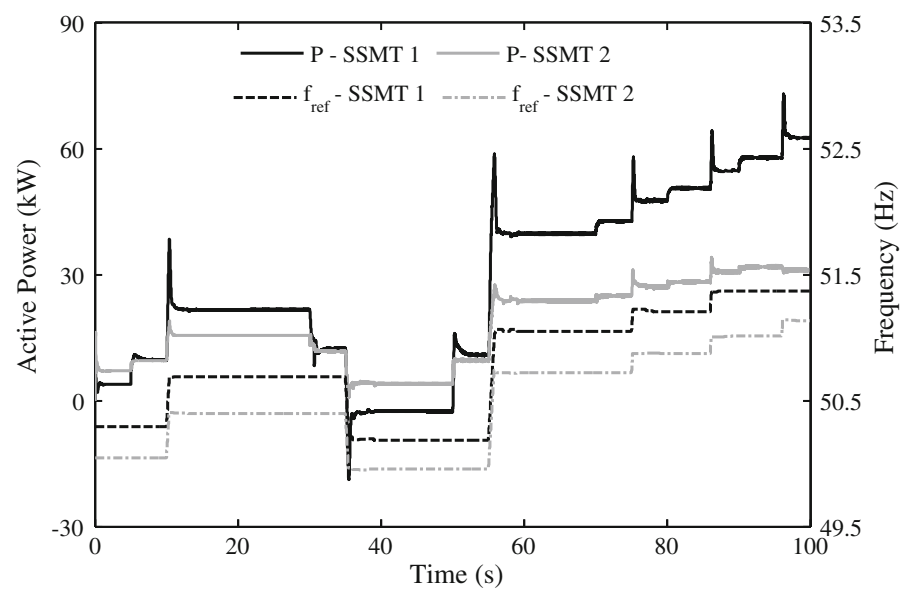

Fig. 12.23 SSMT active power response to the MGCC centralized secondary frequency control

of load there is still sufficient reserve capacity and the MGCC enables the increase of the EV charging power, which is divided in three steps: $20 \%$ of their nominal power at $\mathrm{t}=70 \mathrm{~s}, 40 \%$ at $\mathrm{t}=80 \mathrm{~s}$ and $60 \%$ at $\mathrm{t}=90 \mathrm{~s}$.

As it can be observed in Figs. 12.20 and 12.21, the active participation of the $\mathrm{EV}$ in the frequency regulation reduces the frequency deviation caused by the reconnection of loads and the non-controllable MS. When the frequency deviation falls outside of the P-f droop frequency dead-band (from 49.9 to $50.1 \mathrm{~Hz}$ ), the EV inject power during the reconnection of loads and increase their consumption during the reconnection of the non-controllable MS. The participation of EV in frequency regulation becomes more significant for large frequency disturbances, according to their droop characteristics. Following the MG frequency stabilization $(\mathrm{t}=65 \mathrm{~s})$ and since the MG had enough reserve capacity, the P-f droop parameter $\mathrm{P}_{\text {rated }}$ is increased gradually, in order to allow the EV to charge their batteries. 
Figure 12.22 shows the MG main storage power output for both case studies. As demonstrated, when considering the $\mathrm{V} 2 \mathrm{G}$ operation mode there is a reduction of the active power required from the MG main storage unit.

The active power response of the SSMT to the centralized secondary load frequency control is shown in Fig. 12.23. The SSMT change their power output considering the frequency set-points sent by the MGCC. During the restoration procedure the SSMT coupling inverters are controlled as VSI with a MMO strategy. The DC coupled storage provides immediate power response to the MGCC frequency control signals, compensating the SSMT slower power response.

\subsection{Experimental Validation of MG Emergency Operation}

Following the track record on MG research, significant demonstration activities have been exploited worldwide [15-20]. In Europe, the Association of European Distributed Energy Resources Laboratories (DERlab) clusters European DER laboratories in order to widen the access to the available testing facilities. In the United States, the CERTS Microgrid Laboratory Test Bed aims to demonstrate peer-to-peer and plug-and-play functionalities for different DER, while exploiting the MG islanding operation mode. Large scale demonstration projects can also be found in Japan, with a strong emphasis on the integration of RES in the grid, namely on the development and test of adequate control solutions involving different energy storage technologies to balance the RES variability. Recently, the Zhejiang Electric Power Test and Research Institute implemented a cluster of multiple distributed generation and energy storage technologies capable of operating in different conditions and involving the testing of control strategies for grid connected and islanding mode, while dealing with protection and power quality issues.

Envisioning the development of advanced experimental infrastructures for feasibility demonstration of MG operation in both grid connected and autonomous mode, INESC Porto has been implementing a laboratorial infrastructure in order to exploit distinctive control and management solutions for key DER, such as EV and loads. At the same time, a distinct feature of the MG experimental set-up relies on the integration of both commercially available solutions and in-house developed prototypes. The laboratorial infrastructure aims also to evaluate the specificities of communication requirements for a $\mathrm{MG}$ environment and to assess the impact of different technological solutions on the electric power system operational performance [20]. 

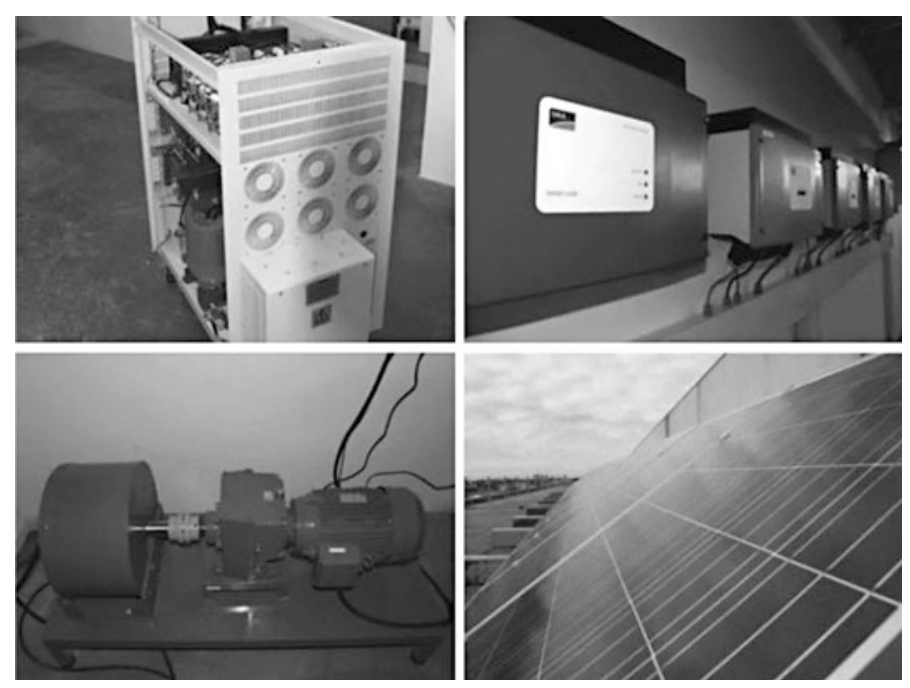

Fig. 12.24 Laboratory renewable energy sources and commercial inverters
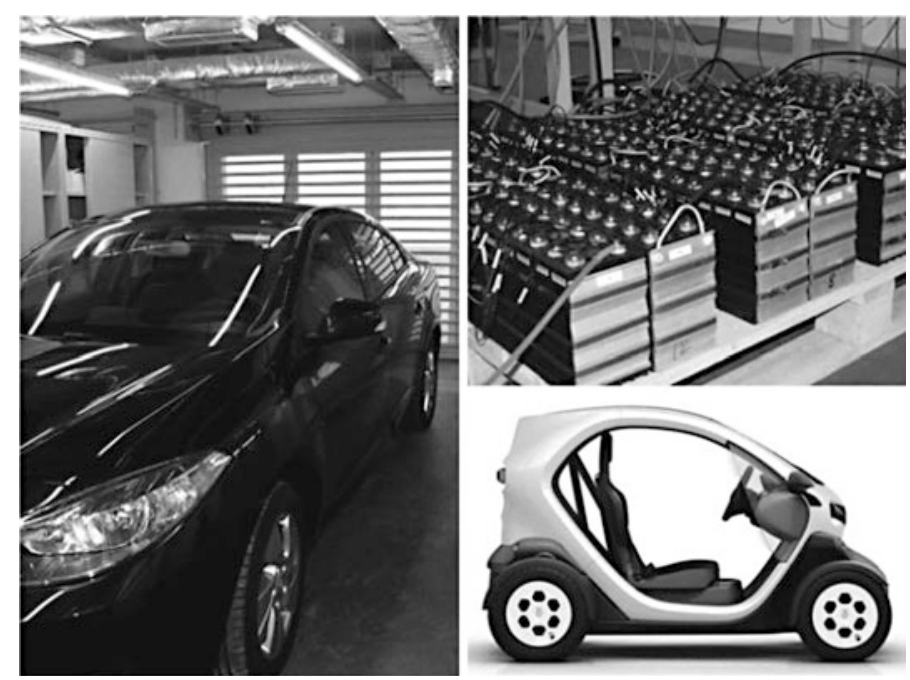

Fig. 12.25 Commercial EV and commercial charger

\subsubsection{MG and EV Laboratory: Electric Infrastructure}

The main building block of this laboratory includes micro-generation technologies, energy storage, controllable loads, EV and the associated chargers as well as the LV grid cable simulators. As shown in Fig. 12.24, the RES based microgeneration 
installed at the laboratory includes $15.5 \mathrm{kWp}$ of photovoltaic installed capacity and a $3 \mathrm{~kW}$ micro-wind turbine emulator. The RES based microgeneration units can be connected to the electric network either through single-phase state of the art commercial inverters or through single-phase inverter prototypes developed in-house. A $20 \mathrm{~kW}$ 4-quadrant back-to-back inverter is also available in the laboratory. The inverter is remotely controlled in terms of injected or absorbed active power, thus allowing the emulation of different operation conditions either as a generation unit or as a load.

Regarding storage units, two Flooded Lead-Acid (FLA) battery banks (50 V, $20 \mathrm{kWh} @ 10 \mathrm{~h}$ ) were integrated in the laboratorial infrastructure, being connected to two three-phase groups of SMA Sunny Islands inverters $(15 \mathrm{~kW}, 400 \mathrm{~V}$ each). These inverters are mainly used for the electrification of remote areas, being able to operate autonomously in isolated systems, by managing storage and local generation (renewable based and/or small backup generators). However, the inverters can also operate in parallel with an existing grid, while providing a smooth transition to autonomous (islanded) operation [40].

Regarding electric mobility, the laboratory has two commercial plug-in EV, as shown in Fig. 12.25, which can be charged by a commercial single-phase home charger. Since the commercial available chargers do not allow controlling the EV charging power, a single-phase $\mathrm{DC} / \mathrm{AC}$ bi-directional inverter prototype was also developed and connected to a bank of lithium-ion batteries (128 cells, $3 \mathrm{~V}$ per cell, $40 \mathrm{Ah})$.

Two three-phase-four wire LV cables emulators (100 and $50 \mathrm{~A}$ ) enable the development of different network testing scenarios, considering the resistive nature of LV feeders. Different unbalanced scenarios can also be implemented, distributing the laboratory electric devices by the three-phases of the system.

All the devices available in the laboratory are connected to an electric panel with three $400 \mathrm{~V}$ busbars with the possibility of being sectionalized (thus allowing a configuration with six semi-busbars). From the installed busbars, a set of output feeders was installed, allowing the connection of the different laboratory equipment. The configuration of the electric panel allows the composition of different MG setups through the use of a contactor based system allowing the selection of the busbar to which a certain feeder should be connected. The laboratory supervision and automation is carried out by a SCADA system, which supports all the laboratorial operations and ensures the electrical network configuration and monitoring. Each feeder and busbar is equipped with a universal metering device, which provides a large set of electrical values and power quality indicators. The interface and field bus capabilities (Modbus) of the universal metering devices enables the communication of measurement data and its incorporation into the laboratory SCADA system, as well as in the software routines used to operate and control the MG system. 


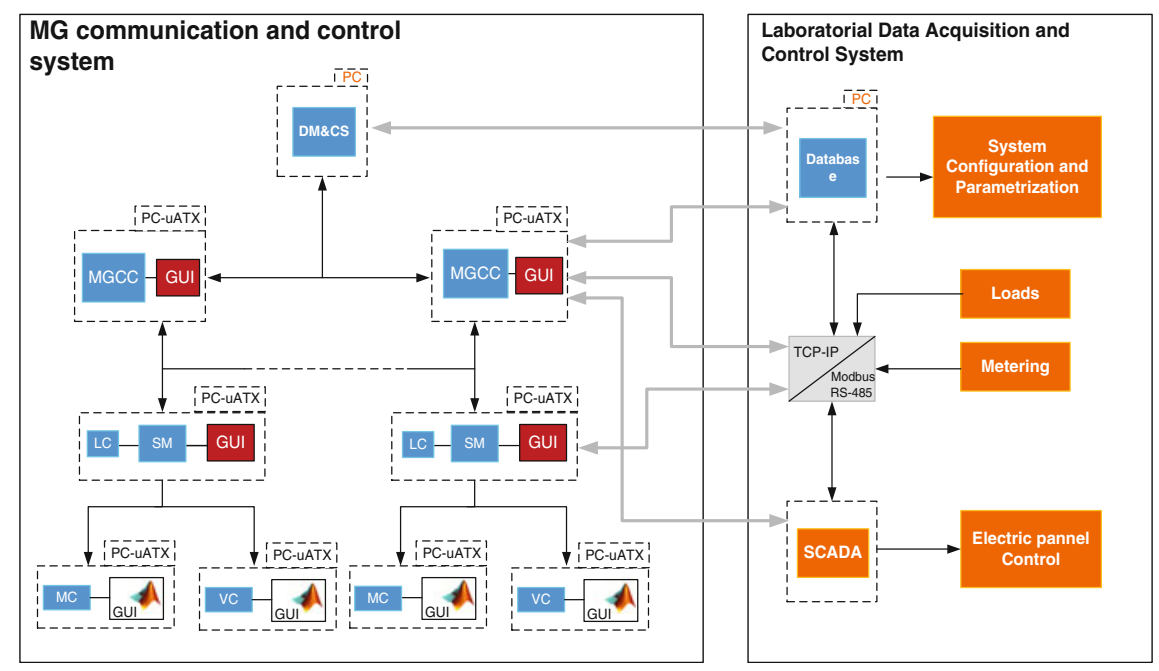

Fig. 12.26 Smart grid laboratorial architecture

\subsubsection{Microgeneration Power Converters and Bi-directional EV Charger Prototypes}

As discussed in Sect. 12.3, the MG management and control strategies have to take into account the specificities of power electronic interfaces coupling microgeneration and EV to the network. In order to develop and test new control strategies and since the commercial inverters are relatively closed technologies, two single-phase microgeneration and an EV charger prototypes were developed [41-43].

The power electronic converters architecture is based on a modular structure, enabling the configuration of different types of converters. The converters are composed by half-bridge assemblies, including IGBT switches and hybrid gate drivers, as well as passive components, such as protection devices, voltage and current sensors and the control hardware.

The inverters prototypes have similar architectures. However, considering their distinct applications they present different control and hardware features. As an illustrative example, the EV bidirectional charger prototype is mentioned. The charger can be divided in two stages with independent control schemes, namely: a grid tied Full-Bridge (FB) inverter that controls the power flow between the DC bus and the LV grid and a Dual Active Bridge (DAB) that regulates the current flowing from/to the batteries and assures the galvanic isolation between the grid and the battery pack. The FB inverter regulates the DC bus voltage to $400 \mathrm{~V}$, in order to ensure adequate supply to the DAB input stage. The inverter is controlled with a PQ strategy within a limit of $\pm 3,680 \mathrm{~W}$, using a proportional-integrative 
controller implemented in a synchronous reference frame and with additional harmonic compensation. The reference power will be determined according to the droop characteristic described in Sect. 12.3.2.

\subsubsection{Communication and Control Architecture}

As represented in Fig. 12.26, the laboratory electric infrastructure is supported by an information and communication system, enabling the management and control of the MG. In terms of communications and control, the laboratory comprises two major building blocks: the MG communication and control system and the laboratory data acquisition and control system. A communication network consisting of an Ethernet infrastructure was deployed and it is used to interlink the previously mentioned building blocks.

At the MG communication and control system, the Ethernet communication system ensures hierarchical and bidirectional communications in line with the MG concept previously discussed and it allows a controllable communication medium interconnecting the different devices and entities (e.g., MGCC, SM, local controllers). Additionally, it was also envisioned the possibility of considering a layer dedicated to the distribution network operator, which is represented by the Distribution Management and Control System (DM\&CS), which will allow the implementation of coordinated operations between the MG cell and the upstream distribution grid.

The MG controllers are emulated in conventional computers integrating the different software and hardware modules responsible for the acquisition and processing of data, which is then used as input to the MG high level management and control functionalities. The information received from the SM includes: power generation, load, EV, responsive loads and power quality indicators, which are processed and aggregated according to the system operator needs. The information received is then used by local software modules, responsible for managing the MG technical operation, during normal and emergency conditions. At the lower control layer, the SM is able to receive set-points from the MGCC and forward them downstream to the respective local controllers. The SM has also a local processing capability, in order to integrate preferences of customers regarding the participation in grid support services and remote management of load and generation.

Regarding the MG communication infrastructure, a Medium Behavior Controller (MBC) was developed. It allows emulating different communication technologies. The Ethernet network is used as a controllable communication medium where distributed MBC are able to impose controlled bandwidth values to the different communication interfaces and define different communication profiles considering data packet delays and losses.

With respect to the laboratory data acquisition and control system, it is intrinsically related to the SCADA system and to the universal metering devices which are used as a platform for data collection for detailed experiment analysis 


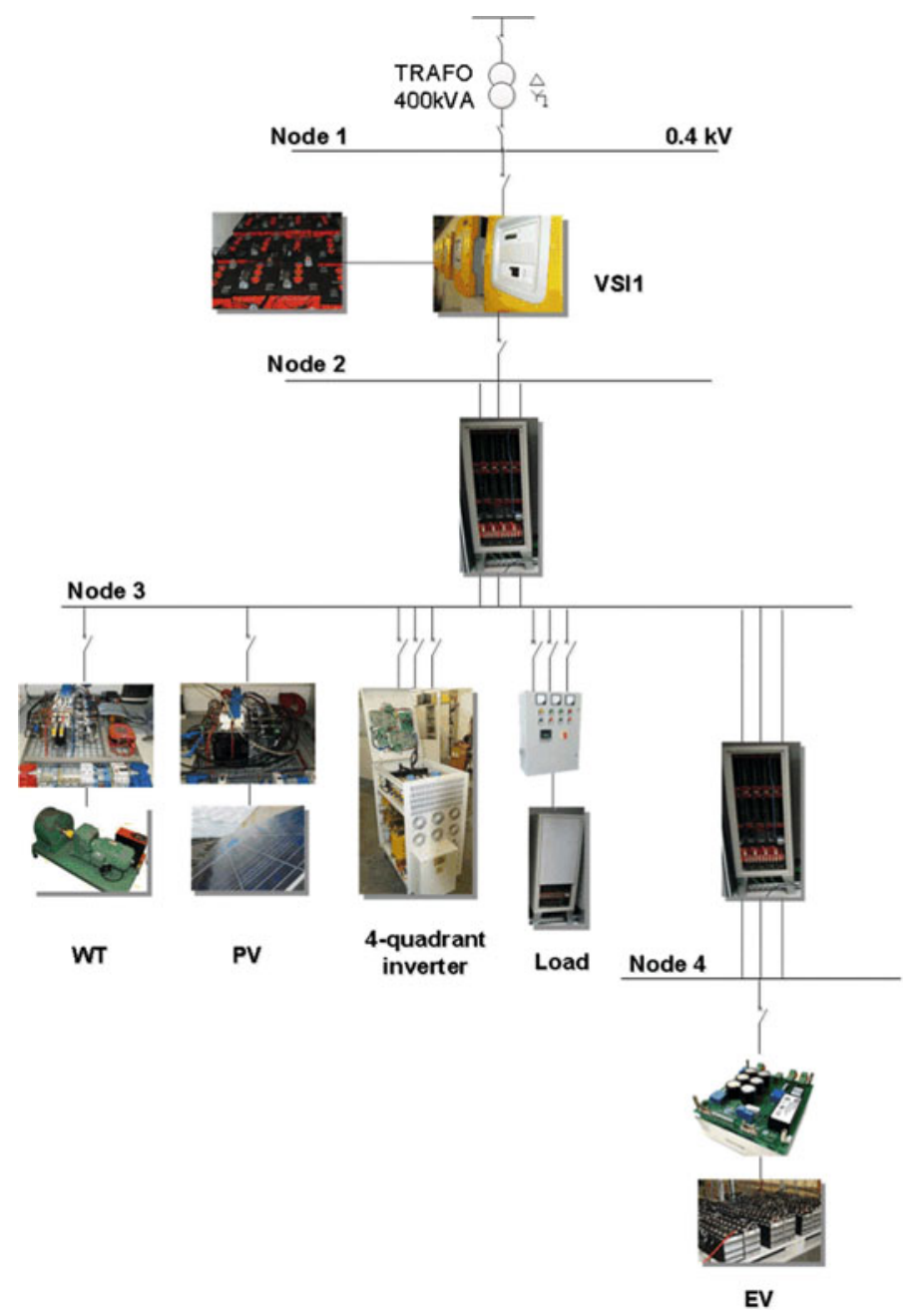

Fig. 12.27 MG test system for MG islanded operation experiment

and to support MG software modules. The Modbus interface of the universal metering devices enables the communication of measurement data and its incorporation into the laboratory SCADA system. Simultaneously, a Modbus to TCP-IP protocol conversion platform is also used in order to share the measurement data with the MG control system and with a general database for storing experimental results. Additionally, laboratory load banks are remotely connected through Ethernet remote I/O devices that allow its remote control and operation. 


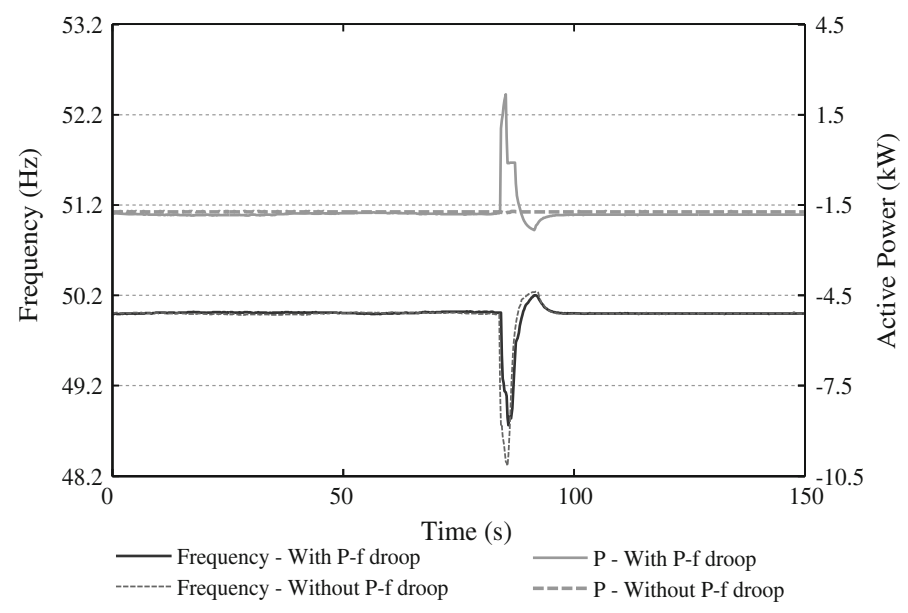

Fig. 12.28 MG frequency and EV active power response to the MG islanding

\subsubsection{Experimental Validation of MG Autonomous Operation}

The experimental MG topology represented in Fig. 12.27 was adopted in order to validate the MG islanded operation. The system includes two PV strings connected to the DC/AC solar power converter prototype, the wind-turbine emulator, the EV bidirectional charger prototype, two $27 \mathrm{~kW}$ resistive banks and the 4-quadrant inverter emulating a gas microturbine with DC coupled storage. The communication architecture, illustrated in Fig. 12.26, was used to support the data control exchanged during the different phases of the scenarios considered.

The three-phase group of VSI inverters-SMA Sunny Island-is connected to node 1 . When operating in normal conditions, loads are supplied directly from the main grid. When the grid switch is disconnected the Sunny island inverter coupled to the FLA storage units ensures power balance and the MG voltage and frequency reference. Primary control is assured by the Sunny Island inverter; however, these power converters intrinsically include a fast additional secondary frequency control strategy, which allows restoring the grid frequency and voltage to the nominal values $[40,44]$. The solar and micro-wind turbine inverter prototypes are operated with fixed power injection, while the 4-quadrant inverter is fully regulated in terms of active power injection. In this case, the 4-quadrant inverter is dispatched to provide secondary regulation, compensating the power injected by the battery bank.

Experiment $1-M G$ islanded operation with EV

As discussed in previous sections, one of the most important features of the MG is the possibility of operating autonomously, through the exploitation of adequate primary and secondary frequency and voltage regulation strategies. Figure 12.28 


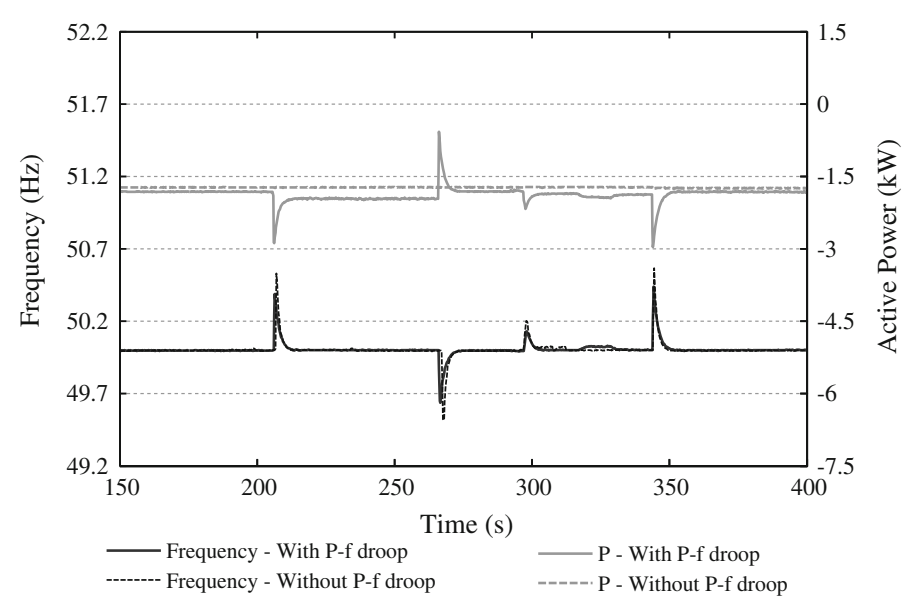

Fig. 12.29 MG frequency and EV active power response during load following

Fig. 12.30 Active power injected by the SMA Sunny Island inverter

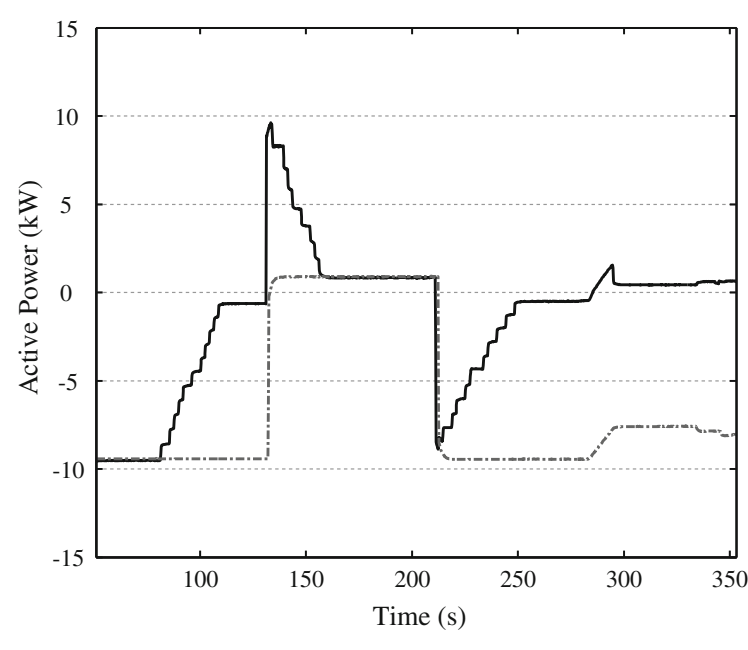

— With Secondary Control -.--.-- Without Secondary Control

compares the MG frequency in the moments subsequent to islanding when considering both the non-controlled and P-f droop EV charging strategies. The MG frequency and EV active power were measured with a power quality analyzer Fluke 1760 (negative values of EV active power corresponds to power absorption for battery charging). Similarly to the results obtained in [4] and [5], the participation of $\mathrm{EV}$ in the $\mathrm{MG}$ frequency regulation reduces the $\mathrm{MG}$ frequency drop during the islanding transient, since it actively responds to the MG frequency by starting to reduce the absorbed power and even reversing it (operation in the V2G 


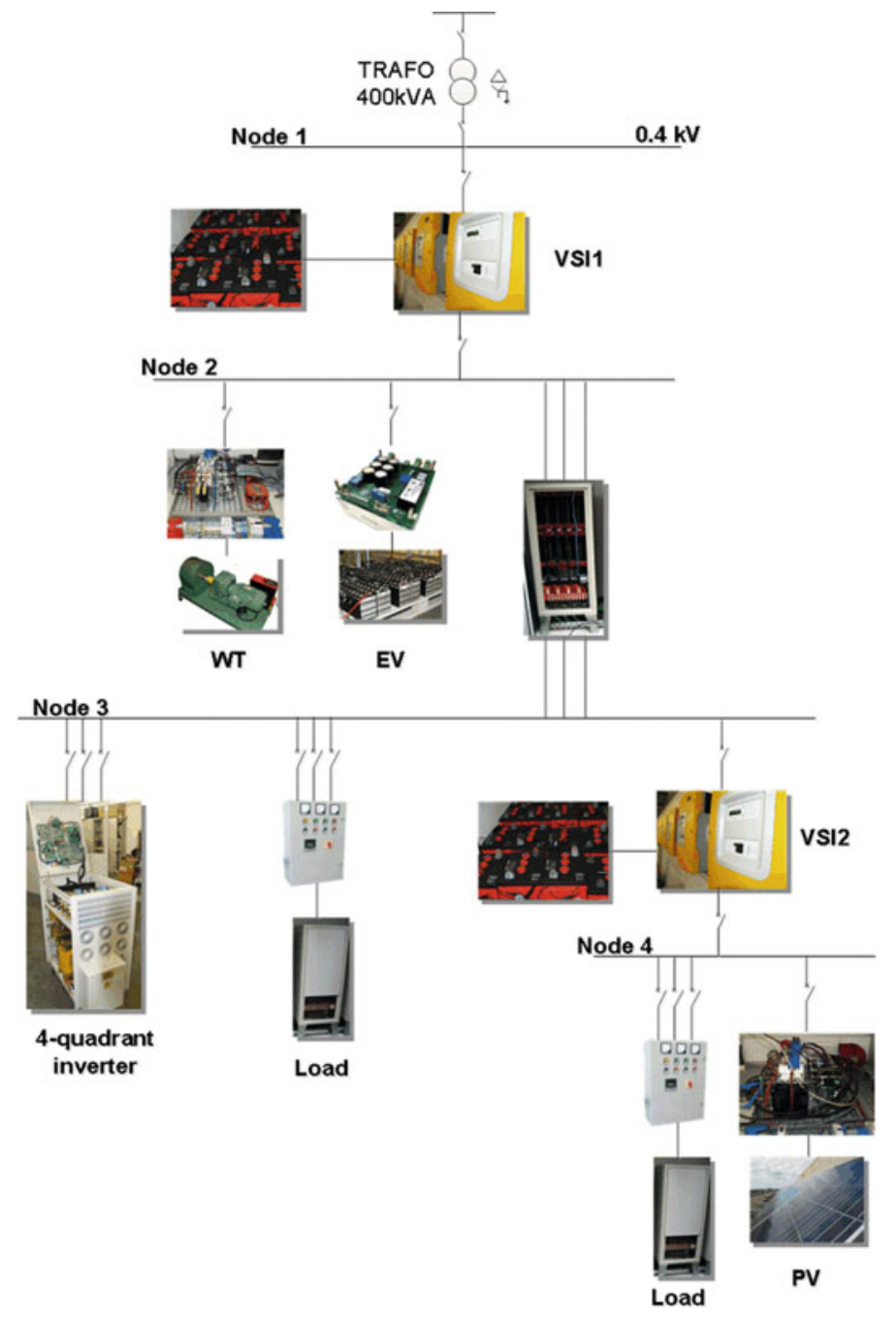

Fig. 12.31 MG test system for the MG restoration procedure

mode). This autonomous behavior implemented in the EV charger prototypes contributes to reduce the power unbalance in the islanded MG and therefore provides an active contribution to $\mathrm{MG}$ frequency regulation.

Following MG islanding, the MG laboratory setup allows also to evaluate the ability of the EV charge prototype to participate in load following conditions. As shown in Fig. 12.29, the EV controlled through P-f droop actively participates in load following during MG islanding operation, by increasing or decreasing its charging power as a response to the different load steps connection (frequency 
decrease) and disconnection (frequency increase). During this process it can also be observed that EV participation is proportional to the MG frequency behavior.

As previously mentioned, SMA Sunny Island inverters are able to provide both primary and secondary frequency regulation. However, after correcting frequency deviation, the inverter continues to supply MG loads if no other MS can be dispatched. In order to compensate the energy provided by the FLA battery bank, the secondary frequency control method described in Sect. 12.3.3 was implemented at the MGCC, which will dispatch the 4-quadrant inverter. Figure 12.30 depicts the active power injected by the SMA Sunny Island inverters both when the secondary regulation functionality is activated and when it is deactivated (negative power refers to power injected from the SMA Sunny Island inverters to the MG). Considering the case without the secondary regulation, in Fig. 12.30 it is possible to observe the active power injection by the SMA Sunny Island inverters starting from an operating conditions which is changed at about $t=140 \mathrm{~s}$ and $\mathrm{t}=210 \mathrm{~s}$ by a load disconnection and reconnection, respectively. When considering the secondary regulation, at about $t=80 \mathrm{~s}$ the MGCC activates secondary control and the 4-quadrant inverter starts increasing its power output, consequently reducing the power injected by the SMA Sunny Island inverters. On the contrary, at $\mathrm{t}=140 \mathrm{~s}$ when there is an excess of generation the 4-quadrant inverter reduces its power output in order to reduce the power absorbed by the SMA Sunny Island inverters.

\section{Experiment $2-M G$ restoration procedure with $E V$}

The MG topology represented in Fig. 12.31 was adopted in order to demonstrate the participation of $\mathrm{EV}$ in the $\mathrm{MG}$ restoration procedure discussed in Sect. 12.4. The test procedure adopted was the following:

1. Before starting the rebuilding phase, the MGCC enables the synchronization of the $\mathrm{EV}$ connected to node 2 to the $\mathrm{MG}$ ( at $\mathrm{t}=25 \mathrm{~s}$ ), in order to provide grid support. As it was previously explained in Sect. 12.4.1, the EV P-f droop characteristic is parameterized by the MGCC with a zero power charging reference.

2. At $t=30 \mathrm{~s}$ the MGCC enables the reconnections of the loads installed in node 3 , representing a total power of $10 \mathrm{~kW}$ and waits until the system frequency is recovered to nominal values.

3. At $\mathrm{t}=44 \mathrm{~s}$ the MGCC enables the reconnection of PV panels connected to node 4.

4. At $\mathrm{t}=56 \mathrm{~s}$ the $\mathrm{MGCC}$ reconnects the controllable generation represented by the 4-quadrant converter connected to node 3 and activates the secondary control.

5. At $\mathrm{t}=80 \mathrm{~s}$, a single-phase (phase A) load step in node 4 is allowed to be connected to the islanded MG, representing a total of $3 \mathrm{~kW}$.

6. Since the system has enough reserve capacity, at $t=136 \mathrm{~s}$ the MGCC enables the increase of the EV charging power in three steps of 20, 40 and $60 \%$ of the EV charging power $(3 \mathrm{~kW})$. 


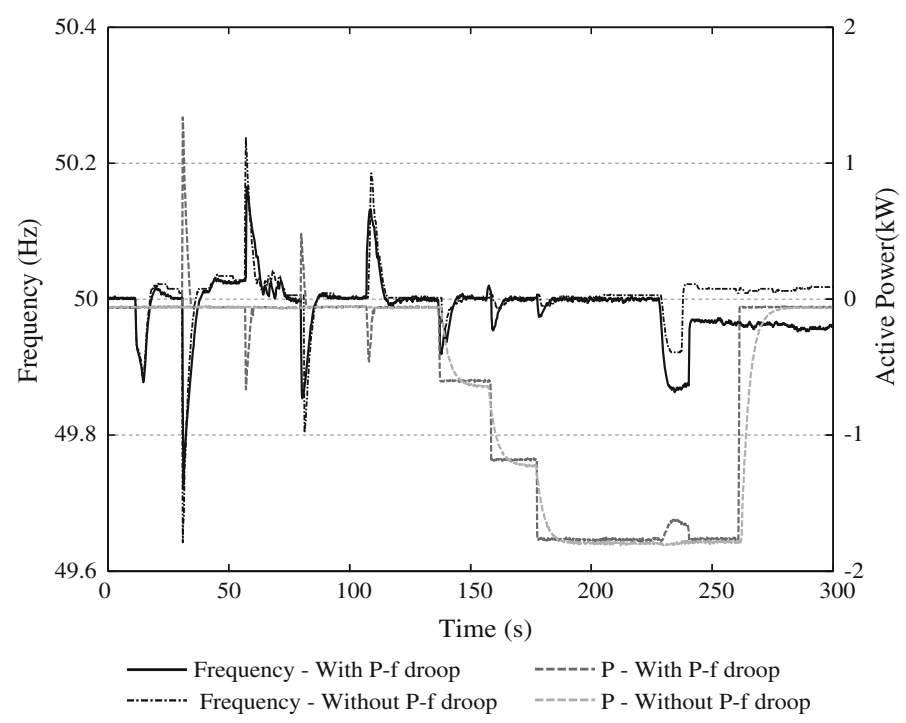

Fig. 12.32 MG frequency response and EV active power during BS with and without P-f droop control

Fig. 12.33 Secondary control response of the 4-Q inverter-delays

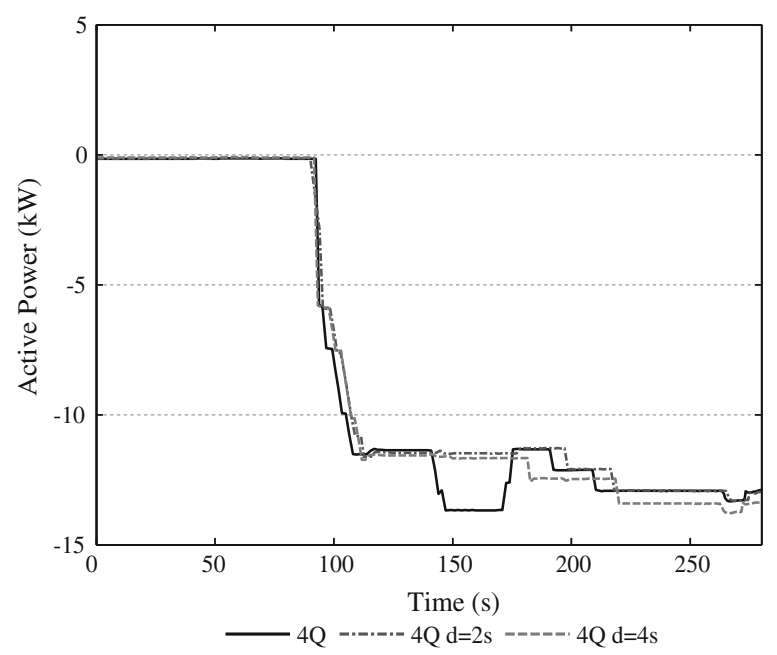

7. At $\mathrm{t}=228 \mathrm{~s}$ the $\mathrm{MV}$ network becomes available and the synchronization process is enabled. Synchronization is performed at the LV side of the MV/LV transformer.

8. At $\mathrm{t}=239 \mathrm{~s}$ the $\mathrm{MG}$ is successfully reconnected to the MV network.

Figure 12.32 compares the MG frequency response and the EV active power output during the restoration procedure (negative power represents EV charger 
Fig. 12.34 Response of the 4-quadrant converterimpact of communication delays and losses

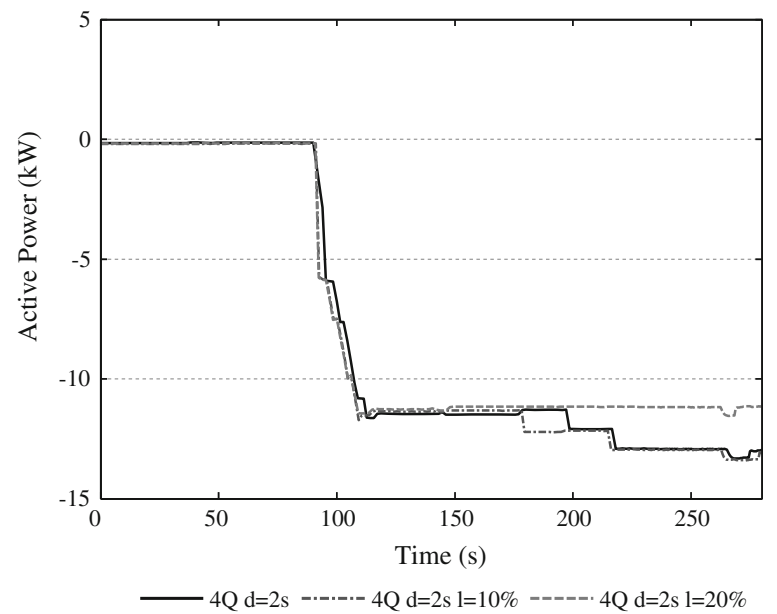

prototype power abortion from the MG). Similarly to the results obtained for the islanded operation, the participation of EV reduces the frequency disturbances resulting from the reconnection of loads and generation. For example, at $\mathrm{t}=30 \mathrm{~s}$ when the load at node 3 is reconnected, the EV active power injection of $1.3 \mathrm{~kW}$ and it contributes to reduce the frequency excursion from 49.64 to $49.73 \mathrm{~Hz}$. On the contrary, when the 4-quadrant converter is reconnected at $t=56 \mathrm{~s}$, the increase of the $\mathrm{EV}$ power consumption to $600 \mathrm{~W}$ reduced the frequency excursion from 50.24 to $50.1 \mathrm{~Hz}$.

In order to evaluate the influence of the communication system uncertainty on the restoration procedure previously developed, the experiment was repeated considering the action of the MBC (described in Sect. 12.5.3) through the emulation of different scenarios of information delays and losses. The introduction of delays and losses is based on probabilistic process over which average values and deviations can be established. Figure 12.33 depict the active power output of the 4-quadrant inverter in the presence of delays associated with the information exchange. When the secondary control is enabled by the MGCC, in this case at $\mathrm{t}=90 \mathrm{~s}$, the secondary control response depends on the MG communication system uncertainties, which are considered to be the delays for information exchange that are changed from a base case (zero delays) to 2 and $4 \mathrm{~s}$ (mean delay values) with a jitter of $500 \mathrm{~ms}$. As shown in Fig. 12.33, an increase in the average delay time, associated with each set-point exchange, delays the overall response of the 4-quadrant converter.

In Fig. 12.34 it is possible to notice the effect of set-point losses over the same experiment, in the particular case of the 4-quadrant converter. A mean delay of approximately $2 \mathrm{~s}$ was considered and different values of information loss were defined. The impact resulting from the communication system uncertainties can be evaluated with the introduction of losses. The impact is visible when comparing the base case with no losses with the remainder cases where losses occur. With a 
loss rate of $10 \%$ a set-point loss occurs around $t=180 \mathrm{~s}$; with a loss rate of $20 \%$, failures are visible $\mathrm{t}=175 \mathrm{~s}$ and $\mathrm{t}=220 \mathrm{~s}$.

\subsection{Final Remarks}

Future power system planning and operation will focus on the LV distribution networks since the majority of small scale DER-EV, MS, storage units and flexible loads - are connected to it. Within the Smart Grid paradigm, the MG concept has been pointed out as an efficient solution to deal with DER integration acting either as a local DER control and management system or as an aggregation cell that is able to provide a coordinate response to the upstream distribution grid.

This new distribution operation philosophy embodies the active distribution network concept, being able to tackle the technical challenges resulting from the large scale integration of DER and EV and at the same time take advantage of the local resources flexibility to improve the security and reliability of the system. The coordination of MG local resources endows the LV system with sufficient autonomy to operate interconnected to the upstream network or islanded, or even perform local restoration procedures in case of generalized disturbances.

Following a worldwide track record on MG research activities, significant demonstrations have been implemented. INESC Porto experience on implementing a MG laboratorial infrastructure was presented aiming the feasibility demonstration of MG operation in grid connected and autonomous mode, while exploiting distinctive control and management solutions for DER. The infrastructure is composed of commercially available components, which are complemented by hardware and software prototypes developed in accordance with strategies developed for DER active integration in MG operation. Additionally, the specificities of communication requirements for a Smart Grid environment and the evaluation of its impact on the MG operational performance, namely due to the associated uncertainty, were addressed given its key relevance for MG operation and control. Furthermore, the presence of distributed control schemes allows the MG to react even when the uncertainty introduced by communication systems is considered.

The industrial development of Smart Grid related products and technologies are growing, enhancing the importance of experimental demonstrations which take into consideration real world conditions. The development of laboratorial facilities, such as the one described in this chapter, plays an important role in the consolidation of innovative solutions that are the key for a successful development of the smart grid paradigm. The results described in this chapter reinforce the fact that the coordination between the MG centralized and local control strategies provides additional resources to deal with the increasing integration of DER. 


\section{References}

1. Lasseter RH (2011) Smart distribution: coupled microgrids. Proc IEEE 99(6):1074-1082

2. Lasseter B (2001) Microgrids [distributed power generation]. IEEE Power Eng Soc Winter Meet 2001 1:146-149

3. Peças Lopes JA, Moreira CL, Madureira AG (2006) Defining control strategies for MicroGrids islanded operation. IEEE Trans Power Syst 21(2):916-924

4. Peças Lopes JA, Silvan Polenz A, Moreira CL, Cherkaoui R (2010) Identification of control and management strategies for LV unbalanced microgrids with plugged-in electric vehicles. Electr Power Syst Res 80(8):898-906

5. Peças Lopes JA, Soares FJ, Almeida PMR (2011) Integration of electric vehicles in the electric power system. Proc IEEE 99(1):168-183

6. Guerrero JM, Vasquez JC, Matas J, de Vicuna LG, Castilla M (2011) Hierarchical control of droop-controlled AC and DC microgrids-a general approach toward standardization. IEEE Trans Industr Electron 58(1):158-172

7. Katiraei F, Iravani MR (2006) Power management strategies for a microgrid with multiple distributed generation units. IEEE Trans Power Syst 21(4):1821-1831

8. Moreira CL, Resende FO, Lopes JAP (2007) Using low voltage microgrids for service restoration. IEEE Trans Power Syst 22(1):395-403

9. Strbac Goran (2008) Demand side management: benefits and challenges. Energy Policy 36(12):4419-4426

10. Schweppe FC, Tabors RD, Kirtley JL, Outhred HR, Pickel FH, Cox AJ (1980) Homeostatic utility control. IEEE Trans Power Apparatus Syst PAS-99(3):1151-1163

11. Short JA, Infield DG, Freris LL (2007) Stabilization of grid frequency through dynamic demand control. IEEE Trans Power Syst 22(3):1284-1293

12. Samarakoon K, Ekanayake J, Jenkins N (2012) Investigation of domestic load control to provide primary frequency response using smart meters. IEEE Trans Smart Grid 3(1):282-292

13. Pourmousavi SA, Nehrir MH (2012) Real-time central demand response for primary frequency regulation in microgrids. IEEE Trans Smart Grid 3(4):1988-1996

14. Gouveia C, Moreira J, Moreira CL, Pecas Lopes JA (2013) Coordinating storage and demand response for microgrid emergency operation. IEEE Trans Smart Grid PP(99):11

15. Hatziargyriou N, Asano H, Iravani R, Marnay C (2007) Microgrids. IEEE Power Energ Mag 5(4):78-94

16. European Distributed Energy Resources Laboratories. http://www.der-lab.net/

17. Lasseter RH, Eto JH, Schenkman B, Stevens J, Vollkommer H, Klapp D, Linton E, Hurtado H, Roy J (2011) CERTS microgrid laboratory test bed. IEEE Trans Power Delivery 26(1):325-332

18. Zhao B, Zhang X, Chen J (2012) Integrated microgrid laboratory system. IEEE Trans Power Syst 27(4):2175-2185

19. Schmitt L, Kumar J, Sun D, Kayal S, Venkata SSM (2013) Ecocity upon a hill: microgrids and the future of the European City. IEEE Power Energ Mag 11(4):59-70

20. Project REIVE - Smart Grids with Electric Vehicles (2013) http://reive.inescporto.pt/en Accessed Jan 2013

21. Shaver D (2009) Low frequency, narrowband PLC standards for smart grid. Presentation

22. Hoch M (2011) Comparison of PLC G3 and PRIME. In: 2011 IEEE international symposium on Power line communications and its applications (ISPLC), pp 165-169, Apr 2011

23. IEEE P1901.2. http://standards.ieee.org/develop/project/1901.2.html

24. IEEE standard for broadband over power line networks: medium access control and physical layer specifications. IEEE Std 1901-2010, pp 1-1586, 2010

25. Parikh PP, Kanabar MG, Sidhu TS (2010) Opportunities and challenges of wireless communication technologies for smart grid applications. In: 2010 IEEE power and energy society general meeting, pp 1-7, Jul 2010 
26. Chin-Sean S, Harada H, Kojima F, Lan Z, Funada R (2011) Smart utility networks in TV white space. IEEE Commun Mag 49(7):132-139

27. IEEE Standard for Local and Metropolitan Area Networks - Part 15.4: Low-Rate Wireless Personal Area Networks (LR-WPANs) Amendment 3: Physical Layer (PHY) Specifications for Low-Data- Rate, Wireless, Smart Metering Utility Networks. IEEE Std 802.15.4 g-2012 (Amendment to IEEE Std 802.15.4-2011), pp 1-252, 272012

28. Gambiroza V, Sadeghi B, Knightly EW (2004) End-to-end performance and fairness in multihop wireless backhaul networks. In: Proceedings of the 10th Annual international conference on mobile computing and networking, mobiCom'04, New York, NY, USA, 2004. ACM, pp 287-301

29. Ernst JB, Denko MK (2011) The design and evaluation of fair scheduling in wireless mesh networks. J Comput Syst Sci 77(4):652-664. JCSS IEEE AINA 2009

30. Gupta GR, Shroff NB (2010) Practical scheduling schemes with throughput guarantees for multi-hop wireless networks. Comput Netw 54(5):766-780

31. Campos R, Duarte R, Sousa F, Ricardo M, Ruela J (2011) Network infrastructure extension using 802.1D-based wireless mesh networks. Wirel Commun Mob Comput 11(1):67-89

32. Campos R, Oliveira C, Ruela J (2011) WiFIX + : a multicast solution for 802.11-based wireless mesh networks. In: 2011 Eighth international conference on wireless on-demand network systems and services (WONS), pp 179-186, Jan 2011

33. Green TC, Prodanovic M (2007) Control of inverter-based micro-grids. Electr Power Syst Res 77(9):1204-1213

34. Blaabjerg F, Teodorescu R, Member SS, Liserre M, Timbus AV (2006) Overview of control and grid synchronization for distributed power generation systems. IEEE Trans Ind Electron 53(5):1398-1409

35. Madureira A, Moreira CL, Peças Lopes JA (2005) Secondary load-frequency control for microgrids in islanded operation. In: Proceedings of ICREPQ'05 - international conference on renewable energies and power quality, Zaragoza, Spain, Março, 2005

36. Jong-Yul K, Jin-Hong J, Seul-Ki K, Changhee C, June Ho P, Hak-Man K, Kee-Young N (2010). Cooperative Control strategy of energy storage system and microsources for stabilizing the microgrid during Islanded operation. IEEE Trans Power Electron 25(12):3037-3048

37. Adibi MM, Fink LH (1994) Power system restoration planning. IEEE Trans Power Syst 9(1):22-28

38. Pham Thi Thu Ha, Besanger Y, Hadjsaid N (2009) New challenges in power system restoration with large scale of dispersed generation insertion. IEEE Trans Power Syst 24(1):398-406

39. Adibi MM, Martins N (2008) Power system restoration dynamics issues. In: 2008 IEEE power and energy society general meeting - conversion and delivery of electrical energy in the 21st Century, pp 1-8

40. SMA Report, "Technology Compedium 2-Solar Stand-Alone Power and Backup Power Supply", SMA 2009. Available at via http://files.sma.de/dl/10040/INSELVERSORAEN101410.pdf

41. Miguel Rodrigues J, Resende F (2012) Using photovoltaic systems to improve voltage control in low voltage networks. In: ISGT2012 - third IEEE PES innovative smart grid technologies Europe conference, Berlin, Germany, Oct 2012

42. Rodrigues J, Resende FO, Moreira CL (2011) Contribution of PMSG based small wind generation systems to provide voltage control in low voltage networks. In: ISGT 2011 - IEEE PES ISGT 2011 Europe, Manchester, UK

43. Varajao D, Araujo RE, Moreira C, Lopes JP (2012) Impact of phase-shift modulation on the performance of a single-stage bidirectional electric vehicle charger. In: IECON 2012 - 38th annual conference on IEEE industrial electronics society, pp 5215-5220, 25-28 Oct 2012

44. Engler A (2005) Applicability of droops in low voltage grids. DER J 1:1-5 